\title{
A FINITE ELEMENT ALGORITHM FOR NEMATIC LIQUID CRYSTAL FLOW BASED ON THE GAUGE-UZAWA METHOD*
}

\author{
Pengzhan Huang ${ }^{1)}$ \\ College of Mathematics and System Sciences, Xinjiang University, Urumqi 830046, China \\ Email: hpzh007@yahoo.com \\ Yinnian $\mathrm{He}$ \\ School of Mathematics and Statistics, Xi'an Jiaotong University, Xi'an 710049, China \\ Email: heyn@mail.xjtu.edu.cn \\ Ting $\mathrm{Li}$ \\ College of Mathematics and System Sciences, Xinjiang University, Urumqi 830046, China \\ Email: 1003887017@qq.com
}

\begin{abstract}
In this paper, we present a finite element algorithm for the time-dependent nematic liquid crystal flow based on the Gauge-Uzawa method. This algorithm combines the Gauge and Uzawa methods within a finite element variational formulation, which is a fully discrete projection type algorithm, whereas many projection methods have been studied without space discretization. Besides, error estimates for velocity and molecular orientation of the nematic liquid crystal flow are shown. Finally, numerical results are given to show that the presented algorithm is reliable and confirm the theoretical analysis.
\end{abstract}

Mathematics subject classification: 65M15, 65M60.

Key words: Nematic liquid crystal model, Finite element approximation, Gauge-Uzawa method, Error analysis.

\section{Introduction}

Given a bounded and convex domain $\Omega \subset \mathbb{R}^{2}$, we consider the following hydrodynamics system modeling the flow of nematic liquid crystal material $[1,18]$

$$
\begin{aligned}
& \mathbf{u}_{t}-\mu \Delta \mathbf{u}+(\mathbf{u} \cdot \nabla) \mathbf{u}+\nabla p+\lambda \nabla \cdot(\nabla \mathbf{b} \odot \nabla \mathbf{b})=\mathbf{f} \\
& \mathbf{b}_{t}-\gamma \Delta \mathbf{b}+(\mathbf{u} \cdot \nabla) \mathbf{b}-\gamma|\nabla \mathbf{b}|^{2} \mathbf{b}=0 \\
& \nabla \cdot \mathbf{u}=0, \quad|\mathbf{b}|=1
\end{aligned}
$$

for $(x, t) \in Q_{T}$, where $Q_{T}=\Omega \times(0, T)$ with a fixed $T \in(0, \infty)$. Here, $\mathbf{u}(x, t): Q_{T} \rightarrow \mathbb{R}^{2}$ and $p(x, t): Q_{T} \rightarrow \mathbb{R}$ denote the velocity field and the pressure of the flow, respectively. Besides, $\mathbf{b}(x, t): Q_{T} \rightarrow \mathbb{S}$ is the director, which represents the molecular orientation field of the nematic liquid crystal material and describes the average molecular alignment, where $\mathbb{S} \subset \mathbb{R}^{2}$ is a unit circle. In addition, $\mathbf{f}(x, t): Q_{T} \rightarrow \mathbb{R}^{2}$ represents a body force on the flow. Three parameters $\mu$, $\lambda$ and $\gamma$ denote the kinematic viscosity, the competition between kinetic and potential energy, and the microscopic elastic relaxation time for the molecular orientation field, respectively. Hereafter, $|\nabla \mathbf{b}|$ or $|\mathbf{b}|$ denotes the Euclidean norm of $\nabla \mathbf{b}$ or $\mathbf{b}$. $\nabla \mathbf{b} \odot \nabla \mathbf{b}$ is an $2 \times 2$ matrix

\footnotetext{
${ }^{*}$ Received January 10, 2020 / Revised version received April 23, 2020 / Accepted May 12, 2020 /

Published online July 14, 2020 /

1) Corresponding author
} 
whose $(i, j)$-the entry is written by $\left(\sum_{k=1}^{2} \frac{\partial b_{k}}{\partial x_{i}} \frac{\partial b_{k}}{\partial x_{j}}\right)_{i, j}$. As in [1], in this paper the system (1.1) is considered in conjunction with the following initial and boundary conditions:

$$
\begin{aligned}
& \mathbf{u}(x, 0)=\mathbf{u}_{0}(x), \quad \mathbf{b}(x, 0)=\mathbf{b}_{0}(x), \quad \forall x \in \Omega, \\
& \left.\mathbf{u}\right|_{S_{T}}=0,\left.\quad \partial_{\mathbf{n}} \mathbf{b}\right|_{S_{T}}=0
\end{aligned}
$$

with $\nabla \cdot \mathbf{u}_{0}=0$ and $\left|\mathbf{b}_{0}\right|=1$, where $S_{T}=\partial \Omega \times(0, T)$ and $\mathbf{n}$ is the outer unit normal of $\partial \Omega$.

The Ericksen-Leslie model, established by Ericksen $[9,10]$ and Leslie [16], can simulate the hydrodynamics of the nematic liquid crystal flows, and it is the macroscopic continuum description of the time evolution of the nematic liquid crystal materials under the influence of both flow velocity and molecular orientation. The system (1.1) was derived by Lin [18] initially as a simplified form of the Ericksen-Leslie model, which is a system of the NavierStokes equations coupled with a convective harmonic map heat flow equation to govern the dynamics of the director field. Although this system neglects the Leslie stress in the EricksenLeslie model, it still retains some essential difficulties of the Ericksen-Leslie model and keeps the core of the mathematical structure, such as strong nonlinearities and constraints, as well as the physical structure, such as the anisotropic effect of elasticity on the velocity field. Thus, the system (1.1) (the simplified Ericksen-Leslie model) can be regarded as a nice initial step towards the theoretical and numerical analysis of the original problem (the Ericksen-Leslie model) [2].

Because of the mathematical and engineering importance of the system (1.1), the simplified Ericksen-Leslie system, there are numerous papers devoted to the theoretical analysis of this system, such as the existence, uniqueness and regularity of solutions. The local existence and uniqueness of strong solutions have been proved by using the standard energy method with rough data [32]. In [15], a blow up criterion has been established for the short time classical solution of the simplified version of Ericksen-Leslie system in two and three dimensions. Hong [14] has given a global existence of solutions to a simplified model of the Ericksen-Leslie system in two dimension with initial data, where the solutions are constructed with at most a finite number of singular times. Both interior and boundary regularity theorems for this system under smallness conditions have been established [19]. Then, the authors have given the existence of global (in time) weak solutions on a bounded smooth domain, which are smooth everywhere with possible exceptions of finitely many singular times. Besides, the global regularity and uniqueness of weak solutions are proved by Xu and Zhang [34] and Lin and Wang [23], respectively.

Since the governing equations (1.1) of the simplified Ericksen-Leslie model include not only the incompressibility, the strong nonlinearity and the physical and nonconvex side constraint $|\mathbf{b}|=1$, but also the coupling between the harmonic map flow and the fluid equations of motion, which make it not easy to solve these equations effectively. Therefore, much effort has been throwing to the development of some efficient numerical methods for investigating this problem.

On one hand, in order to weaken the nonconvex side constraint, a well-known penalty formulation for (1.1) called the Ginzburg-Landau function is studied by Lin and Liu [20]. In fact, Liu and Walkington [21] initially considered the numerical approximation of this Ginzburg-Landau penalized problem in two-dimensional domains. Further, to eliminate the need of Hermite finite elements, the same authors have constructed some mixed approximations where derivatives of the director field are approximated "independently" of the director [22]. A fully discrete mixed scheme, based on continuous finite elements in space and a linear semi-implicit first-order integration in time, has been shown in [12]. Besides, Cabrales et al. [6,7] have presented a projection-based time-splitting algorithm, where the velocity and pressure are computed by using a projection-based algorithm and the director is computed jointly to an auxiliary vari- 
able. An accurate and efficient Legendre-Galerkin method which can preserve energy law in discrete form has been designed [35]. Du et al. [8] have studied a Fourier-spectral method for the considered system. The spectral accuracy of this method has established. Further, a linear fully discrete mixed scheme has been considered in [11] using finite element method in space and a semi-implicit Euler scheme in time.

On other hand, instead of applying the Ginzburg-Landau function to weaken the nonconvex side constraint, there are many works which directly approximate the simplified Ericksen-Leslie system (1.1). Becker et al. [4] have constructed a fully discrete scheme, which used some low order finite elements and enjoyed a discrete energy law. A fully splitting and decoupled in time linear algorithm has been designed for the simplified Ericksen-Leslie system with explicit treatment of the unitary constraint for the director field by an augmented Lagrangian technique [13]. Besides, An and Su [1] have shown optimal error estimates for an linearized semi-implicit Euler finite element scheme for the considered system. The first constraint-preserving, decoupled and energy-stable scheme has been constructed by Bao et al. [3].

Unlike the above methods for the simplified Ericksen-Leslie system (1.1), a finite element algorithm based on the Gauge-Uzawa method $[24,25]$ is considered in this paper, which does not require an artificial boundary condition on pressure and avoids dealing with boundary derivatives, compared to the gauge method and the pressure-correction projection method for incompressible flows. Besides, an optimal error estimate has obtained via energy estimate for the semi-discrete Gauge-Uzawa method of the Navier-Stokes equations [26]. Then, Pyo has designed second-order time discrete schemes of the Gauge-Uzawa method for the NavierStokes equations [27] and the Boussinesq equations [28], based on a second order backward difference formula. Furthermore, for the incompressible viscous flows with variable density [29], the viscoelastic Oldroyd flows [30], the conduction-convection equations [31], the incompressible magnetohydrodynamics equations [36] and the incompressible natural convection problem with variable density [33], the efficiency and validity of the Gauge-Uzawa method are shown.

In this paper, inspired by [24,25], we will study a finite element algorithm based on the Gauge-Uzawa method to solve the simplified Ericksen-Leslie system (1.1). Compared to the previous model using the Gauge-Uzawa method, the current model has stronger nonlinearity and physical constraint. Besides, due to the complexity of the current model, we improve numerical analysis of the Gauge-Uzawa method in the proof and use mathematical induction method. The paper is organized as follows. In Section 2, we will introduce some notations for the nematic liquid crystal model, and construct the Gauge-Uzawa finite element algorithm for the system (1.1). In the next section, we give the error analysis for velocity and director. Then, in Section 4, numerical results are presented to confirm our theoretical analysis.

\section{A Finite Element Algorithm Based on the Gauge-Uzawa Method}

In order to introduce a finite element discretization of the system (1.1) based on the GaugeUzawa method, the further notation will be needed.

For the mathematical setting of the problem (1.1) with the boundary and initial conditions (1.2), we introduce the usual $L^{2}(\Omega)$ norm and its inner product by $\|\cdot\|_{0}$ and $(\cdot, \cdot)$, respectively. Denote by $L_{0}^{2}(\Omega)$ the subspace of $L^{2}(\Omega)$ of function with vanishing mean value. The $L^{p}(\Omega)$ norm and $W^{m, p}(\Omega)$ norm are denoted by $\|\cdot\|_{L^{p}(\Omega)}$ and $\|\cdot\|_{W^{m, p}(\Omega)}$, respectively, for $m \in \mathbb{N}^{+}$, $1 \leq p \leq \infty$. In particular, $H^{m}(\Omega)$ is used to represent the space $W^{m, 2}(\Omega)$ and $\|\cdot\|_{m}$ denotes the norm in $H^{m}(\Omega)$. 
Then we define the following particular subspaces of $H^{1}(\Omega)^{2}$ :

$$
\mathbf{V}=H_{0}^{1}(\Omega)^{2}=\left\{\mathbf{v} \in H^{1}(\Omega)^{d}:\left.\mathbf{v}\right|_{\partial \Omega}=0\right\}
$$

Besides, for $X$ being a normed function space in $\Omega, L^{p}(0, T ; X)$ is the space of all functions defined on $Q_{T}$ for which the norm

$$
\|u\|_{L^{p}(0, T ; X)}=\left(\int_{0}^{T}\|u\|_{X}^{p} \mathrm{dt}\right)^{\frac{1}{p}}, \quad p \in[1, \infty),
$$

is finite.

Furthermore, let $\Im=\{K\}$ be a shape-regular quasi-uniform partition of $\Omega$ of meshsize $h$ into closed elements $K$. Now, set $\hat{b} \in H_{0}^{1}(K)$ take the value 1 at the barycenter of $K$ and satisfy that $0 \leq \hat{b}(\hat{x}) \leq 1$, which is called a "bubble function". Next, we introduce the following finite element discrete subspaces for (1.1) as in [2]

$$
\begin{aligned}
& \mathbb{V}_{h}:=\left\{\mathbf{v}_{h} \in \mathbf{V} \cap C^{0}(\Omega)^{2}:\left.\mathbf{v}_{h}\right|_{K} \in\left(P_{1}(K) \oplus \operatorname{span}\{\hat{b}\}\right)^{2} \quad \forall K \in \Im\right\}, \\
& \mathbb{T}_{h}:=\left\{\mathbf{r}_{h} \in C^{0}(\Omega)^{2}:\left.\mathbf{r}_{h}\right|_{K} \in P_{1}(K)^{2} \quad \forall K \in \Im\right\}, \\
& \mathbb{P}_{h}:=\left\{q_{h} \in L_{0}^{2}(\Omega) \cap C^{0}(\Omega):\left.q_{h}\right|_{K} \in P_{1}(K) \quad \forall K \in \Im\right\},
\end{aligned}
$$

where $P_{1}(K)$ is the set of all polynomials on $K$ of degree no more than 1 . Next, let $N>0$ be a fixed integer number and $\left\{t_{n}\right\}_{n=0}^{N}$ be a uniform partition of $[0, T]$ and $t_{n}=n \tau$ with time step $\tau=T / N$.

In addition, we need the following trilinear form

$$
\Pi_{h}\left(\mathbf{u}_{h}, \mathbf{v}_{h}, \mathbf{w}_{h}\right)=\frac{1}{2}\left(\left(\mathbf{u}_{h} \cdot \nabla\right) \mathbf{v}_{h}, \mathbf{w}_{h}\right)-\frac{1}{2}\left(\left(\mathbf{u}_{h} \cdot \nabla\right) \mathbf{w}_{h}, \mathbf{v}_{h}\right),
$$

which satisfies following property [24]

$$
\Pi_{h}\left(\mathbf{u}_{h}, \mathbf{v}, \mathbf{w}_{h}\right) \leq C\left\|\mathbf{u}_{h}\right\|_{0}\|\mathbf{v}\|_{2}\left\|\nabla \mathbf{w}_{h}\right\|_{0} \quad \forall \mathbf{u}_{h}, \mathbf{w}_{h} \in \mathbb{V}_{h}, \mathbf{v} \in H^{2}(\Omega)^{2} .
$$

Here and after, we denote $C$ (with or without a subscript) a general positive constant which is independent of $h$ and $\tau$ and may stand for different values at different occurrences. In addition, we need the following inverse inequality [5], which holds for $\mathbf{v}_{h} \in \mathbb{V}_{h}$ :

$$
\left\|\mathbf{v}_{h}\right\|_{W^{l, p}(\Omega)^{2}} \leq C h^{m-l+2 \min \left\{0, \frac{1}{p}-\frac{1}{q}\right\}}\left\|\mathbf{v}_{h}\right\|_{W^{m, q}(\Omega)^{2}}, \quad 1 \leq p, q \leq \infty, \quad 0 \leq m \leq l .
$$

Let $\phi$ be an gauge variable [24] and $\mathbf{a}$ be a function such that $\mathbf{u}=\mathbf{a}+\nabla \phi$. If $\phi$ and $p$ satisfy equation $\phi_{t}-\nu \Delta \phi+p=0$, then (1.1) can be rewritten as

$$
\begin{aligned}
& \mathbf{a}_{t}-\mu \Delta \mathbf{a}+(\mathbf{u} \cdot \nabla) \mathbf{u}+\lambda \nabla \cdot(\nabla \mathbf{b} \odot \nabla \mathbf{b})=\mathbf{f} \\
& \mathbf{b}_{t}-\gamma \Delta \mathbf{b}+(\mathbf{u} \cdot \nabla) \mathbf{b}-\gamma|\nabla \mathbf{b}|^{2} \mathbf{b}=0 \\
& \nabla \cdot \mathbf{a}+\Delta \phi=0, \quad|\mathbf{b}|=1
\end{aligned}
$$

Consequently, start with $\phi^{0}=0$ and $\mathbf{a}^{0}=\mathbf{u}^{0}$. Repeat

$$
\begin{aligned}
& \tau^{-1}\left(\mathbf{a}^{n+1}-\mathbf{a}^{n}\right)-\mu \Delta \mathbf{a}^{n+1}+\left(\mathbf{u}^{n} \cdot \nabla\right)\left(\mathbf{a}^{n+1}+\nabla \phi^{n}\right)+\lambda \nabla \cdot\left(\nabla \mathbf{b}^{n} \odot \nabla \mathbf{b}^{n}\right)=\mathbf{f}\left(t_{n+1}\right), \\
& \tau^{-1}\left(\mathbf{b}^{n+1}-\mathbf{b}^{n}\right)-\gamma \Delta \mathbf{b}^{n+1}+\left(\mathbf{u}^{n+1} \cdot \nabla\right) \mathbf{b}^{n+1}-\gamma\left|\nabla \mathbf{b}^{n}\right|^{2} \mathbf{b}^{n}=0, \\
& \nabla \cdot \mathbf{a}^{n+1}+\Delta \phi^{n+1}=0, \quad\left|\mathbf{b}^{n+1}\right|=1 .
\end{aligned}
$$


Moreover, set $\widehat{\mathbf{u}}^{n+1}=\mathbf{a}^{n+1}+\nabla \phi^{n}$. Inserting it into (2.3) yields

$$
\begin{aligned}
& \tau^{-1}\left(\widehat{\mathbf{u}}^{n+1}-\mathbf{u}^{n}\right)-\mu \Delta \widehat{\mathbf{u}}^{n+1}+\left(\mathbf{u}^{n} \cdot \nabla\right) \widehat{\mathbf{u}}^{n+1}+\mu \nabla s^{n}+\lambda \nabla \cdot\left(\nabla \mathbf{b}^{n} \odot \nabla \mathbf{b}^{n}\right)=\mathbf{f}\left(t_{n+1}\right), \\
& \tau^{-1}\left(\mathbf{b}^{n+1}-\mathbf{b}^{n}\right)-\gamma \Delta \mathbf{b}^{n+1}+\left(\mathbf{u}^{n+1} \cdot \nabla\right) \mathbf{b}^{n+1}-\gamma\left|\nabla \mathbf{b}^{n}\right|^{2} \mathbf{b}^{n}=0 \\
& \nabla \cdot \widehat{\mathbf{u}}^{n+1}+\Delta \rho^{n+1}=0, \quad\left|\mathbf{b}^{n+1}\right|=1,
\end{aligned}
$$

where $s^{n}=\Delta \phi^{n}$ and $\rho^{n+1}=\phi^{n+1}-\phi^{n}$.

We are now prepared to give a finite element discretization of the system (1.1) based on the Gauge-Uzawa method.

Algorithm 2.1. Let $s_{h}^{0}=0$ and $\mathbf{u}_{h}^{0} \in \mathbb{V}_{h}, \mathbf{b}_{h}^{0} \in \mathbb{T}_{h}$ be the solutions of $\left(\mathbf{u}_{h}^{0}, \mathbf{w}_{h}\right)=\left(\mathbf{u}_{0}, \mathbf{w}_{h}\right)$ and $\left(\mathbf{b}_{h}^{0}, \mathbf{r}_{h}\right)=\left(\mathbf{b}_{0}, \mathbf{r}_{h}\right)$ for all $\mathbf{w}_{h} \in \mathbb{V}_{h}, \mathbf{r}_{h} \in \mathbb{T}_{h}$, respectively.

Step 1. Find $\widehat{\mathbf{u}}_{h}^{n+1} \in \mathbb{V}_{h}$ as the solution of

$$
\begin{aligned}
& \tau^{-1}\left(\widehat{\mathbf{u}}_{h}^{n+1}-\mathbf{u}_{h}^{n}, \mathbf{w}_{h}\right)+\Pi_{h}\left(\mathbf{u}_{h}^{n}, \widehat{\mathbf{u}}_{h}^{n+1}, \mathbf{w}_{h}\right)+\mu\left(\nabla \widehat{\mathbf{u}}_{h}^{n+1}, \nabla \mathbf{w}_{h}\right)-\mu\left(s_{h}^{n}, \nabla \cdot \mathbf{w}_{h}\right) \\
& =\left(\mathbf{f}\left(t_{n+1}\right), \mathbf{w}_{h}\right)+\lambda\left(\nabla \mathbf{b}_{h}^{n} \odot \nabla \mathbf{b}_{h}^{n}, \nabla \mathbf{w}_{h}\right), \quad \forall \mathbf{w}_{h} \in \mathbb{V}_{h} .
\end{aligned}
$$

Step 2. Find $\rho_{h}^{n+1} \in \mathbb{P}_{h}$ as the solution of

$$
\left(\nabla \rho_{h}^{n+1}, \nabla \psi_{h}\right)=\left(\nabla \cdot \widehat{\mathbf{u}}_{h}^{n+1}, \psi_{h}\right), \quad \forall \psi_{h} \in \mathbb{P}_{h} .
$$

Step 3. Update $s_{h}^{n+1} \in \mathbb{P}_{h}$ based on

$$
\left(s_{h}^{n+1}, q_{h}\right)=\left(s_{h}^{n}, q_{h}\right)-\left(\nabla \cdot \widehat{\mathbf{u}}_{h}^{n+1}, q_{h}\right), \quad \forall q_{h} \in \mathbb{P}_{h} .
$$

Step 4. IUpdate $\mathbf{u}_{h}^{n+1}$ based on

$$
\mathbf{u}_{h}^{n+1}=\widehat{\mathbf{u}}_{h}^{n+1}+\nabla \rho_{h}^{n+1} .
$$

Step 5. Find $\mathbf{b}_{h}^{n+1} \in \mathbb{T}_{h}$ as the solution of

$$
\begin{aligned}
& \tau^{-1}\left(\mathbf{b}_{h}^{n+1}-\mathbf{b}_{h}^{n}, \mathbf{r}_{h}\right)+\Pi_{h}\left(\mathbf{u}_{h}^{n+1}, \mathbf{b}_{h}^{n+1}, \mathbf{r}_{h}\right)+\gamma\left(\nabla \mathbf{b}_{h}^{n+1}, \nabla \mathbf{r}_{h}\right) \\
& -\gamma\left(\left|\nabla \mathbf{b}_{h}^{n}\right|^{2} \mathbf{b}_{h}^{n}, \mathbf{r}_{h}\right)=0, \quad \forall \mathbf{r}_{h} \in \mathbb{T}_{h} .
\end{aligned}
$$

Remark 2.1. Based on (2.7), for all $\psi_{h} \in \mathbb{P}_{h}$,

$$
\left(\mathbf{u}_{h}^{n+1}, \nabla \psi_{h}\right)=\left(\widehat{\mathbf{u}}_{h}^{n+1}, \nabla \psi_{h}\right)+\left(\nabla \rho_{h}^{n+1}, \nabla \psi_{h}\right)=0,
$$

where we have used (2.5). Thus, $\mathbf{u}_{h}^{n+1}$ is weakly discrete divergence free while $\widehat{\mathbf{u}}_{h}^{n+1}$ is not, based on (2.5).

\section{Error Estimates}

We are now in a position to state and prove error estimates for the finite element algorithm for nematic liquid crystal flow based on the gauge-Uzawa method. In the following error esti- 
mates, we always assume that the solutions of (1.1) satisfy the following regularity assumptions:

$$
\begin{array}{lll}
\mathbf{u} \in L^{\infty}\left(0, T ; H^{2}(\Omega)^{2}\right), & \mathbf{u}_{t} \in L^{2}\left(0, T ; H^{1}(\Omega)^{2}\right), & \mathbf{u}_{t t} \in L^{2}\left(0, T ; L^{2}(\Omega)^{2}\right), \\
\mathbf{b} \in L^{\infty}\left(0, T ; H^{2}(\Omega)^{2}\right), & \mathbf{b}_{t} \in L^{2}\left(0, T ; H^{1}(\Omega)^{2}\right), & \mathbf{b}_{t t} \in L^{2}\left(0, T ; L^{2}(\Omega)^{2}\right) .
\end{array}
$$

We begin the analysis by introducing a sequence of Stokes equations as follows: For $n \in$ $[0, N-1]$, find $\left(\mathbf{U}^{n+1}, P^{n+1}\right) \in \mathbf{V} \times L_{0}^{2}(\Omega)$ to yield

$$
\begin{aligned}
& \tau^{-1}\left(\mathbf{U}^{n+1}-\mathbf{U}^{n}\right)-\mu \Delta \mathbf{U}^{n+1}+\nabla P^{n+1} \\
& \quad=\mathbf{f}\left(t_{n+1}\right)-((\mathbf{u} \cdot \nabla) \mathbf{u})\left(t_{n+1}\right)-(\lambda \nabla \cdot(\nabla \mathbf{b} \odot \nabla \mathbf{b}))\left(t_{n+1}\right), \\
& \nabla \cdot \mathbf{U}^{n+1}=0
\end{aligned}
$$

where $\mathbf{U}^{0}=\mathbf{u}_{0}$.

Firstly, for the sake of simplicity, we denote errors $\mathbf{G}^{n+1}=\mathbf{u}\left(t_{n+1}\right)-\mathbf{U}^{n+1}$ and $g^{n+1}=$ $p\left(t_{n+1}\right)-P^{n+1}$.

In fact, subtracting (3.2) from (1.1) at $t=t_{n+1}$, we have

$$
\begin{aligned}
& \tau^{-1}\left(\mathbf{G}^{n+1}-\mathbf{G}^{n}\right)-\mu \Delta \mathbf{G}^{n+1}+\nabla g^{n+1}=-\tau^{-1} \int_{t_{n}}^{t_{n+1}}\left(t-t_{n}\right) \mathbf{u}_{t t} \mathrm{dt} \\
& \nabla \cdot \mathbf{G}^{n+1}=0 .
\end{aligned}
$$

Then, we have the following variational formulation of the problem $(3.3)$ : Find $\left(\mathbf{G}^{n+1}, g^{n+1}\right) \in$ $\mathbf{V} \times L_{0}^{2}(\Omega)$ such that, for all $(\mathbf{w}, q) \in \mathbf{V} \times L_{0}^{2}(\Omega)$

$$
\begin{aligned}
& \tau^{-1}\left(\mathbf{G}^{n+1}-\mathbf{G}^{n}, \mathbf{w}\right)-\mu\left(\nabla \mathbf{G}^{n+1}, \nabla \mathbf{w}\right)-\left(\nabla \cdot \mathbf{w}, g^{n+1}\right) \\
& \quad=-\tau^{-1} \int_{t_{n}}^{t_{n+1}}\left(t-t_{n}\right)\left(\mathbf{u}_{t t}, \mathbf{w}\right) \mathrm{dt}, \\
& \left(\nabla \cdot \mathbf{G}^{n+1}, q\right)=0 .
\end{aligned}
$$

Setting $\mathbf{w}=2 \tau \mathbf{G}^{n+1}$ and $q=2 \tau g^{n+1}$ and adding the ensuing equations, we deduce that

$$
\begin{aligned}
& \left\|\mathbf{G}^{n+1}\right\|_{0}^{2}-\left\|\mathbf{G}^{n}\right\|_{0}^{2}+\left\|\mathbf{G}^{n+1}-\mathbf{G}^{n}\right\|_{0}^{2}+2 \tau \mu\left\|\nabla \mathbf{G}^{n+1}\right\|_{0}^{2} \\
\leq & \tau \mu\left\|\nabla \mathbf{G}^{n+1}\right\|_{0}^{2}+C \tau^{2} \int_{t_{n}}^{t_{n+1}}\left\|\mathbf{u}_{t t}\right\|_{0}^{2} \mathrm{dt} .
\end{aligned}
$$

Then, sum (3.5) with respect to $n$ from 0 to $N-1$ to get

$$
\left\|\mathbf{G}^{N}\right\|_{0}^{2}+\sum_{n=0}^{N-1}\left\|\mathbf{G}^{n+1}-\mathbf{G}^{n}\right\|_{0}^{2}+\tau \mu \sum_{n=0}^{N-1}\left\|\nabla \mathbf{G}^{n+1}\right\|_{0}^{2} \leq C \tau^{2}\left\|\mathbf{u}_{t t}\right\|_{L^{2}\left(0, T ; L^{2}(\Omega)^{2}\right)}^{2} .
$$

Further, divide both sides of (3.4) by $\|\nabla \mathbf{w}\|$ and take the supremum over $\mathbf{w} \in \mathbf{V}$. This gives

$$
\left\|g^{n+1}\right\|_{0}^{2} \leq C\left(\tau^{-2}\left\|\mathbf{G}^{n+1}-\mathbf{G}^{n}\right\|_{0}^{2}+\left\|\nabla \mathbf{G}^{n+1}\right\|_{0}^{2}+\tau\left\|\mathbf{u}_{t t}\right\|_{L^{2}\left(t_{n}, t_{n+1} ; L^{2}(\Omega)^{2}\right)}^{2}\right) .
$$

Hence, multiplying (3.7) by $\tau$ and summing the ensuing equation with respect to $n$ from 0 to $N-1$, we arrive at

$$
\tau \sum_{n=0}^{N-1}\left\|g^{n+1}\right\|_{0}^{2} \leq C\left(\tau^{-1} \sum_{n=0}^{N-1}\left\|\mathbf{G}^{n+1}-\mathbf{G}^{n}\right\|_{0}^{2}+\tau \sum_{n=0}^{N-1}\left\|\nabla \mathbf{G}^{n+1}\right\|_{0}^{2}+\tau^{2}\right) \leq C \tau .
$$

Then, based on (3.8) and (3.6), we have the following lemma. 
Lemma 3.1. Under the assumptions of (3.1), we have

$$
\begin{aligned}
& \left\|\boldsymbol{G}^{N}\right\|_{0}^{2}+\sum_{n=0}^{N-1}\left\|\boldsymbol{G}^{n+1}-\boldsymbol{G}^{n}\right\|_{0}^{2}+\mu \tau \sum_{n=0}^{N-1}\left\|\nabla \boldsymbol{G}^{n+1}\right\|_{0}^{2} \leq C \tau^{2}, \\
& \tau \sum_{n=0}^{N-1}\left\|g^{n+1}\right\|_{0}^{2} \leq C \tau .
\end{aligned}
$$

Secondly, as [24], we define $\left(\mathbf{U}_{h}^{n+1}, P_{h}^{n+1}\right)=\mathcal{G}_{h}\left(\mathbf{u}\left(t_{n+1}\right), p\left(t_{n+1}\right)\right) \in \mathbb{V}_{h} \times \mathbb{P}_{h}$ to be the Stokes projection of the exact solution at time $t_{n+1}$. Next, we denote errors $\mathbf{G}_{h}^{n+1}=\mathbf{u}\left(t_{n+1}\right)-\mathbf{U}_{h}^{n+1}$ and $g_{h}^{n+1}=p\left(t_{n+1}\right)-P_{h}^{n+1}$. Further, we have the following bounds.

Lemma 3.2 ([24]). Under the assumptions of (3.1), we get

$$
\left\|\boldsymbol{G}_{h}^{n+1}\right\|_{0}+h\left\|\nabla \boldsymbol{G}_{h}^{n+1}\right\|_{0}+h\left\|g_{h}^{n+1}\right\|_{0} \leq C h^{2} .
$$

In fact, denote $\mathbf{F}^{n+1}=\mathbf{U}^{n+1}-\mathbf{U}_{h}^{n+1}$ and $f^{n+1}=P^{n+1}-P_{h}^{n+1}$. Then, combining Lemma 3.2 and 3.1 leads to

$$
\begin{aligned}
& \tau \sum_{n=0}^{N-1}\left\|\nabla \mathbf{F}^{n+1}\right\|_{0}^{2} \leq C\left(\tau^{2}+h^{2}\right), \\
& \left\|\mathbf{F}^{N}\right\|_{0}+\tau \sum_{n=0}^{N-1}\left\|f^{n+1}\right\|_{0}^{2} \leq C\left(\tau+h^{2}\right) .
\end{aligned}
$$

Moreover, let $\mathbf{b}\left(t_{n+1}\right)$ be the true solution of the director at time $t=t_{n+1}$ and $\mathbf{b}_{h}\left(t_{n+1}\right) \in \mathbb{T}_{h}$ be its $H^{1}$-projection $[17,25]$, namely,

$$
\left(\nabla \mathbf{b}_{h}\left(t_{n+1}\right), \nabla \mathbf{r}_{h}\right)=\left(\nabla \mathbf{b}\left(t_{n+1}\right), \nabla \mathbf{r}_{h}\right), \quad \forall \mathbf{r}_{h} \in \mathbb{T}_{h}
$$

Defining $\boldsymbol{\vartheta}^{n+1}:=\mathbf{b}\left(t_{n+1}\right)-\mathbf{b}_{h}\left(t_{n+1}\right)$, and using interpolation theory, we have $[17,25]$

$$
\left\|\boldsymbol{\vartheta}^{n+1}\right\|_{0}+h\left\|\nabla \boldsymbol{\vartheta}^{n+1}\right\|_{0} \leq C h^{2} .
$$

Thirdly, we denote errors $\mathbf{E}^{n+1}=\mathbf{U}^{n+1}-\mathbf{u}_{h}^{n+1}, \widehat{\mathbf{E}}^{n+1}=\mathbf{U}^{n+1}-\widehat{\mathbf{u}}_{h}^{n+1}, e^{n+1}=P^{n+1}-p_{h}^{n+1}$ and $\varepsilon^{n+1}=\mathbf{b}\left(t_{n+1}\right)-\mathbf{b}_{h}^{n+1}$. Similarly, denote errors $\mathbf{E}_{h}^{n+1}=\mathbf{U}_{h}^{n+1}-\mathbf{u}_{h}^{n+1}, \widehat{\mathbf{E}}_{h}^{n+1}=\mathbf{U}_{h}^{n+1}-\widehat{\mathbf{u}}_{h}^{n+1}$, $e_{h}^{n+1}=P_{h}^{n+1}-p_{h}^{n+1}$ and $\varepsilon_{h}^{n+1}=\mathbf{b}_{h}\left(t_{n+1}\right)-\mathbf{b}_{h}^{n+1}$.

Then, in order to obtain the error equations, subtract (2.4) from (3.2) to obtain

$$
\begin{aligned}
& \tau^{-1}\left(\widehat{\mathbf{E}}^{n+1}-\mathbf{E}^{n}, \mathbf{w}_{h}\right)+\mu\left(\nabla \widehat{\mathbf{E}}^{n+1}, \nabla \mathbf{w}_{h}\right)+\Pi_{h}\left(\mathbf{u}\left(t_{n+1}\right), \mathbf{u}\left(t_{n+1}\right), \mathbf{w}_{h}\right)-\Pi_{h}\left(\mathbf{u}_{h}^{n}, \widehat{\mathbf{u}}_{h}^{n+1}, \mathbf{w}_{h}\right) \\
= & \left(P^{n+1}, \nabla \cdot \mathbf{w}_{h}\right)-\mu\left(s_{h}^{n}, \nabla \cdot \mathbf{w}_{h}\right)+\lambda\left(\nabla \mathbf{b}\left(t_{n+1}\right) \odot \nabla \mathbf{b}\left(t_{n+1}\right)-\nabla \mathbf{b}_{h}^{n} \odot \nabla \mathbf{b}_{h}^{n}, \nabla \mathbf{w}_{h}\right) .
\end{aligned}
$$

Besides, rewrite (1.1) at $t=t_{n+1}$ as follows:

$$
\begin{aligned}
& \tau^{-1}\left(\mathbf{b}\left(t_{n+1}\right)-\mathbf{b}\left(t_{n}\right), \mathbf{r}\right)+\gamma\left(\nabla \mathbf{b}\left(t_{n+1}\right), \nabla \mathbf{r}\right)+\Pi_{h}\left(\mathbf{u}\left(t_{n+1}\right), \mathbf{b}\left(t_{n+1}\right), \mathbf{r}\right) \\
= & \gamma\left(\left|\nabla \mathbf{b}\left(t_{n+1}\right)\right|^{2} \mathbf{b}\left(t_{n+1}\right), \mathbf{r}\right)+\tau^{-1} \int_{t_{n}}^{t_{n+1}}\left(t-t_{n}\right)\left(\mathbf{b}_{t t}, \mathbf{r}\right) \mathrm{dt} .
\end{aligned}
$$

Then, subtracting (2.8) from (3.12) with $\mathbf{r}=\mathbf{r}_{h}$, we have

$$
\begin{aligned}
& \tau^{-1}\left(\varepsilon^{n+1}-\varepsilon^{n}, \mathbf{r}_{h}\right)+\gamma\left(\nabla \varepsilon^{n+1}, \nabla \mathbf{r}_{h}\right)+\Pi_{h}\left(\mathbf{u}\left(t_{n+1}\right), \mathbf{b}\left(t_{n+1}\right), \mathbf{r}_{h}\right)-\Pi_{h}\left(\mathbf{u}_{h}^{n+1}, \mathbf{b}_{h}^{n+1}, \mathbf{r}_{h}\right) \\
= & \gamma\left(\left|\nabla \mathbf{b}\left(t_{n+1}\right)\right|^{2} \mathbf{b}\left(t_{n+1}\right), \mathbf{r}_{h}\right)-\gamma\left(\left|\nabla \mathbf{b}_{h}^{n}\right|^{2} \mathbf{b}_{h}^{n}, \mathbf{r}_{h}\right)+\tau^{-1} \int_{t_{n}}^{t_{n+1}}\left(t-t_{n}\right)\left(\mathbf{b}_{t t}, \mathbf{r}_{h}\right) \mathrm{dt} .
\end{aligned}
$$

We are ready to state the main results of this section. 
Theorem 3.1. Under the assumptions of $(3.1)$, if $\tau=\mathcal{O}\left(h^{2}\right)$ is valid, then we have

$$
\begin{aligned}
& \left\|\boldsymbol{u}(T)-\boldsymbol{u}_{h}^{N}\right\|_{0}^{2}+\tau \mu \sum_{n=0}^{N-1}\left\|\nabla\left(\boldsymbol{u}\left(t_{n+1}\right)-\boldsymbol{u}_{h}^{n+1}\right)\right\|_{0}^{2} \\
& \quad+\left\|\boldsymbol{b}(T)-\boldsymbol{b}_{h}^{N}\right\|_{0}^{2}+\tau \gamma \sum_{n=0}^{N-1}\left\|\nabla\left(\boldsymbol{b}\left(t_{n+1}\right)-\boldsymbol{b}_{h}^{n+1}\right)\right\|_{0}^{2} \\
& \leq C\left(\tau+h^{2}\right) .
\end{aligned}
$$

Proof. On one hand, set $\mathbf{w}_{h}=2 \tau \widehat{\mathbf{E}}_{h}^{n+1}$ in (3.11), and note that $\widehat{\mathbf{E}}_{h}^{n+1}=\widehat{\mathbf{E}}^{n+1}-\mathbf{F}^{n+1}$ and $\widehat{\mathbf{E}}^{n+1}=\mathbf{E}^{n+1}+\nabla \rho_{h}^{n+1}$. Then

$$
\begin{aligned}
& \tau^{-1}\left(\widehat{\mathbf{E}}^{n+1}-\mathbf{E}^{n}, 2 \tau \widehat{\mathbf{E}}_{h}^{n+1}\right)=2\left(\mathbf{E}^{n+1}+\nabla \rho_{h}^{n+1}-\mathbf{E}^{n}, \mathbf{E}^{n+1}+\nabla \rho_{h}^{n+1}-\mathbf{F}^{n+1}\right) \\
= & \left\|\mathbf{E}^{n+1}\right\|_{0}^{2}-\left\|\mathbf{E}^{n}\right\|_{0}^{2}+\left\|\mathbf{E}^{n+1}-\mathbf{E}^{n}\right\|_{0}^{2}-2\left(\mathbf{E}^{n+1}-\mathbf{E}^{n}, \mathbf{F}^{n+1}\right)+2\left\|\nabla \rho_{h}^{n+1}\right\|_{0}^{2},
\end{aligned}
$$

as well as

$$
\begin{aligned}
\mu\left(\nabla \widehat{\mathbf{E}}^{n+1}, 2 \tau \nabla \widehat{\mathbf{E}}_{h}^{n+1}\right) & =2 \mu \tau\left(\nabla \widehat{\mathbf{E}}^{n+1}, \nabla\left(\widehat{\mathbf{E}}^{n+1}-\mathbf{F}^{n+1}\right)\right) \\
& =2 \mu \tau\left\|\nabla \widehat{\mathbf{E}}^{n+1}\right\|_{0}^{2}-2 \mu \tau\left(\nabla \mathbf{E}^{n+1}, \nabla \mathbf{F}^{n+1}\right)
\end{aligned}
$$

Hence, combining (3.15) and (3.14), we have

$$
\begin{aligned}
& \left\|\mathbf{E}^{n+1}\right\|_{0}^{2}-\left\|\mathbf{E}^{n}\right\|_{0}^{2}+\left\|\mathbf{E}^{n+1}-\mathbf{E}^{n}\right\|_{0}^{2}+2 \mu \tau\left\|\nabla \widehat{\mathbf{E}}^{n+1}\right\|_{0}^{2}+2\left\|\nabla \rho_{h}^{n+1}\right\|_{0}^{2} \\
= & 2\left(\mathbf{E}^{n+1}-\mathbf{E}^{n}, \mathbf{F}^{n+1}\right)+2 \mu \tau\left(\nabla \widehat{\mathbf{E}}^{n+1}, \nabla \mathbf{F}^{n+1}\right)+2 \tau\left(P^{n+1}, \nabla \cdot \widehat{\mathbf{E}}_{h}^{n+1}\right) \\
& \quad-2 \mu \tau\left(s_{h}^{n}, \nabla \cdot \widehat{\mathbf{E}}_{h}^{n+1}\right)-2 \tau\left(\Pi_{h}\left(\mathbf{u}\left(t_{n+1}\right), \mathbf{u}\left(t_{n+1}\right), \widehat{\mathbf{E}}_{h}^{n+1}\right)-\Pi_{h}\left(\mathbf{u}_{h}^{n}, \widehat{\mathbf{u}}_{h}^{n+1}, \widehat{\mathbf{E}}_{h}^{n+1}\right)\right) \\
& \quad+2 \tau \lambda\left(\nabla \mathbf{b}\left(t_{n+1}\right) \odot \nabla \mathbf{b}\left(t_{n+1}\right)-\nabla \mathbf{b}_{h}^{n} \odot \nabla \mathbf{b}_{h}^{n}, \nabla \widehat{\mathbf{E}}_{h}^{n+1}\right) \\
= & : \sum_{i=1}^{6} A_{i} .
\end{aligned}
$$

Now, we estimate each term of $A_{i}$ separately. By using the Cauchy-Schwarz and Young inequality, we have

$$
\begin{aligned}
& A_{1} \leq \frac{1}{2}\left\|\mathbf{E}^{n+1}-\mathbf{E}^{n}\right\|_{0}^{2}+C\left\|\mathbf{F}^{n+1}\right\|_{0}^{2}, \\
& A_{2} \leq \frac{\mu \tau}{8}\left\|\nabla \widehat{\mathbf{E}}^{n+1}\right\|_{0}^{2}+C \mu \tau\left\|\nabla \mathbf{F}^{n+1}\right\|_{0}^{2} .
\end{aligned}
$$

Besides, by employing $\widehat{\mathbf{E}}_{h}^{n+1}=\mathbf{E}_{h}^{n+1}+\nabla \rho_{h}^{n+1}$ which results from (2.7), it follows from Lemma 3.1 that

$$
\begin{aligned}
A_{3}= & 2 \tau\left(f^{n+1}-g_{h}^{n+1}+p\left(t_{n+1}\right), \nabla \cdot \widehat{\mathbf{E}}_{h}^{n+1}\right)=2 \tau\left(f^{n+1}, \nabla \cdot \widehat{\mathbf{E}}_{h}^{n+1}\right) \\
& \quad+2 \tau\left(\nabla g_{h}^{n+1}, \nabla \rho_{h}^{n+1}\right)-2 \tau\left(\nabla p\left(t_{n+1}\right), \nabla \rho_{h}^{n+1}\right)+2 \tau\left(P_{h}^{n+1}, \nabla \cdot \mathbf{E}_{h}^{n+1}\right) \\
\leq & \frac{\mu \tau}{8}\left(\left\|\nabla \widehat{\mathbf{E}}^{n+1}\right\|_{0}^{2}+\left\|\nabla \mathbf{F}^{n+1}\right\|_{0}^{2}\right)+C \tau\left\|f^{n+1}\right\|_{0}^{2}+C \tau^{2}+\left\|\nabla \rho_{h}^{n+1}\right\|_{0}^{2},
\end{aligned}
$$

where we have noticed the fact that $\left(P_{h}^{n+1}, \nabla \cdot \mathbf{E}_{h}^{n+1}\right)=0$. 
Consequently, in view of (2.6), we deduce that $\left\|s_{h}^{n+1}-s_{h}^{n}\right\|_{0}=\left\|\nabla \cdot \widehat{\mathbf{u}}_{h}^{n+1}\right\|_{0} \leq\left\|\nabla \widehat{\mathbf{E}}^{n+1}\right\|_{0}$. In what follows, we arrive at

$$
\begin{aligned}
A_{4} & \leq-2 \tau\left(s_{h}^{n}, \nabla \cdot\left(\mathbf{U}_{h}^{n+1}-\widehat{\mathbf{u}}_{h}^{n+1}\right)\right) \\
& \leq 2 \tau\left(s_{h}^{n}, \nabla \cdot \widehat{\mathbf{u}}_{h}^{n+1}\right)=2 \mu \tau\left(s_{h}^{n}-s_{h}^{n+1}, s_{h}^{n}\right) \\
& =\mu \tau\left(\left\|s_{h}^{n}\right\|_{0}^{2}-\left\|s_{h}^{n+1}\right\|_{0}^{2}+\left\|s_{h}^{n}-s_{h}^{n+1}\right\|_{0}^{2}\right) \\
& \leq \mu \tau\left\|s_{h}^{n}\right\|_{0}^{2}-\left\|s_{h}^{n+1}\right\|_{0}^{2}+\mu \tau\left\|\nabla \widehat{\mathbf{E}}^{n+1}\right\|_{0}^{2} .
\end{aligned}
$$

In order to estimate $A_{5}$, we add and subtract $\Pi_{h}\left(\mathbf{u}\left(t_{n}\right), \mathbf{u}\left(t_{n+1}\right), \widehat{\mathbf{E}}_{h}^{n+1}\right)$ and note that $\Pi_{h}\left(\mathbf{u}_{h}^{n}\right.$, $\left.\widehat{\mathbf{E}}_{h}^{n+1}, \widehat{\mathbf{E}}_{h}^{n+1}\right)=0$. Then

$$
\begin{aligned}
A_{5}=-2 \tau & \Pi_{h}\left(\mathbf{u}\left(t_{n+1}\right)-\mathbf{u}\left(t_{n}\right), \mathbf{u}\left(t_{n+1}\right), \widehat{\mathbf{E}}_{h}^{n+1}\right)-2 \tau \Pi_{h}\left(\mathbf{u}\left(t_{n}\right)-\mathbf{u}_{h}^{n}, \mathbf{u}\left(t_{n+1}\right), \widehat{\mathbf{E}}_{h}^{n+1}\right) \\
& \quad-2 \tau \Pi_{h}\left(\mathbf{u}_{h}^{n}, \mathbf{u}\left(t_{n+1}\right)-\widehat{\mathbf{u}}_{h}^{n+1}, \widehat{\mathbf{E}}_{h}^{n+1}\right) \\
= & -2 \tau \Pi_{h}\left(\mathbf{u}\left(t_{n+1}\right)-\mathbf{u}\left(t_{n}\right), \mathbf{u}\left(t_{n+1}\right), \widehat{\mathbf{E}}_{h}^{n+1}\right)-2 \tau \Pi_{h}\left(\mathbf{G}^{n}+\mathbf{E}^{n}, \mathbf{u}\left(t_{n+1}\right), \widehat{\mathbf{E}}_{h}^{n+1}\right) \\
& \quad-2 \tau \Pi_{h}\left(\mathbf{u}\left(t_{n}\right), \mathbf{G}_{h}^{n+1}, \widehat{\mathbf{E}}_{h}^{n+1}\right)+2 \tau \Pi_{h}\left(\mathbf{G}^{n}+\mathbf{E}^{n}, \mathbf{G}_{h}^{n+1}, \widehat{\mathbf{E}}_{h}^{n+1}\right) \\
& \quad: \sum_{i=1}^{4} B_{i} .
\end{aligned}
$$

According to (2.1) and Young inequality, we have

$$
\begin{aligned}
B_{1} & \leq C \tau\left\|\mathbf{u}\left(t_{n+1}\right)-\mathbf{u}\left(t_{n}\right)\right\|_{0}\left\|\mathbf{u}\left(t_{n+1}\right)\right\|_{2}\left\|\nabla \widehat{\mathbf{E}}_{h}^{n+1}\right\|_{0} \\
& \leq \frac{\mu \tau}{64}\left\|\nabla \widehat{\mathbf{E}}_{h}^{n+1}\right\|_{0}^{2}+C \tau^{2}\left\|\mathbf{u}_{t}(t)\right\|_{L^{2}\left(t^{n}, t^{n+1} ; L^{2}(\Omega)^{2}\right)} \\
B_{2} & \leq C \tau\left\|\mathbf{G}^{n}+\mathbf{E}^{n}\right\|_{0}\left\|\mathbf{u}\left(t_{n+1}\right)\right\|_{2}\left\|\nabla \widehat{\mathbf{E}}_{h}^{n+1}\right\|_{0} \\
& \leq \frac{\mu \tau}{64}\left\|\nabla \widehat{\mathbf{E}}_{h}^{n+1}\right\|_{0}^{2}+C \tau\left(\left\|\mathbf{G}^{n}\right\|_{0}^{2}+\left\|\mathbf{E}^{n}\right\|_{0}^{2}\right), \\
B_{3} & \leq C \tau\left\|\mathbf{u}\left(t_{n}\right)\right\|_{2}\left\|\mathbf{G}_{h}^{n+1}\right\|_{0}\left\|\nabla \widehat{\mathbf{E}}_{h}^{n+1}\right\|_{0} \leq \frac{\mu \tau}{64}\left\|\nabla \widehat{\mathbf{E}}_{h}^{n+1}\right\|_{0}^{2}+C \tau\left\|\mathbf{G}_{h}^{n+1}\right\|_{0}^{2},
\end{aligned}
$$

as well as

$$
\begin{aligned}
B_{4} \leq C \tau\left\|\mathbf{G}^{n}+\mathbf{E}^{n}\right\|_{0}\left\|\mathbf{G}_{h}^{n+1}\right\|_{L^{\infty}(\Omega)^{2}}\left\|\nabla \widehat{\mathbf{E}}_{h}^{n+1}\right\|_{0}+C \tau \| \mathbf{G}^{n} \\
\quad+\mathbf{E}^{n}\left\|_{0}\right\| \mathbf{G}_{h}^{n+1}\left\|_{W^{1,3}(\Omega)^{2}}\right\| \widehat{\mathbf{E}}_{h}^{n+1} \|_{L^{6}(\Omega)^{2}} \\
\leq C \tau\left\|\mathbf{G}^{n}+\mathbf{E}^{n}\right\|_{0}\left(h^{-1}\left\|\mathbf{G}_{h}^{n+1}\right\|_{0}+\left\|\nabla \mathbf{G}_{h}^{n+1}\right\|_{0}\right)\left\|\nabla \widehat{\mathbf{E}}_{h}^{n+1}\right\|_{0} \\
\leq \frac{\mu \tau}{64}\left\|\nabla \widehat{\mathbf{E}}_{h}^{n+1}\right\|_{0}^{2}+C \tau h\left(\left\|\mathbf{G}^{n}\right\|_{0}^{2}+\left\|\mathbf{E}^{n}\right\|_{0}^{2}\right),
\end{aligned}
$$

where we have employed the inverse inequality (2.2) and Lemma 3.2. Thus, combining these $B_{i}$ and noticing that $(a+b)^{2} \leq 2\left(a^{2}+b^{2}\right)$, we get the bound of $A_{5}$.

$$
\begin{aligned}
A_{5} & \leq \frac{\mu \tau}{16}\left\|\nabla \widehat{\mathbf{E}}_{h}^{n+1}\right\|_{0}^{2}+C \tau^{2}\left\|\mathbf{u}_{t}(t)\right\|_{L^{2}\left(t^{n}, t^{n+1} ; L^{2}(\Omega)^{2}\right)}+C \tau\left(\left\|\mathbf{G}^{n}\right\|_{0}^{2}+\left\|\mathbf{E}^{n}\right\|_{0}^{2}+h^{4}\right) \\
& \leq \frac{\mu \tau}{8}\left\|\nabla \widehat{\mathbf{E}}^{n+1}\right\|_{0}^{2}+\frac{\mu \tau}{8}\left\|\nabla \mathbf{F}^{n+1}\right\|_{0}^{2}+C \tau\left(\left\|\mathbf{G}^{n}\right\|_{0}^{2}+\left\|\mathbf{E}^{n}\right\|_{0}^{2}+h^{4}\right) .
\end{aligned}
$$


For the last term $A_{6}$, we rewrite it as follows

$$
\begin{aligned}
A_{6}=2 & \lambda\left(\nabla \mathbf{b}\left(t_{n+1}\right) \odot \nabla\left(\mathbf{b}\left(t_{n+1}\right)-\mathbf{b}\left(t_{n}\right)\right), \nabla \widehat{\mathbf{E}}_{h}^{n+1}\right) \\
& +2 \tau \lambda\left(\nabla\left(\mathbf{b}\left(t_{n+1}\right)-\mathbf{b}\left(t_{n}\right)\right) \odot \nabla \mathbf{b}\left(t_{n}\right), \nabla \widehat{\mathbf{E}}_{h}^{n+1}\right) \\
& +2 \tau \lambda\left(\nabla\left(\mathbf{b}\left(t_{n}\right)-\mathbf{b}_{h}^{n}\right) \odot \nabla \mathbf{b}\left(t_{n}\right), \nabla \widehat{\mathbf{E}}_{h}^{n+1}\right)+2 \tau \lambda\left(\nabla \mathbf{b}_{h}^{n} \odot \nabla\left(\mathbf{b}\left(t_{n}\right)-\mathbf{b}_{h}^{n}\right), \nabla \widehat{\mathbf{E}}_{h}^{n+1}\right) \\
=2 & \lambda\left(\nabla \mathbf{b}\left(t_{n+1}\right) \odot \nabla\left(\mathbf{b}\left(t_{n+1}\right)-\mathbf{b}\left(t_{n}\right)\right), \nabla \widehat{\mathbf{E}}_{h}^{n+1}\right) \\
& +2 \tau \lambda\left(\nabla\left(\mathbf{b}\left(t_{n+1}\right)-\mathbf{b}\left(t_{n}\right)\right) \odot \nabla \mathbf{b}\left(t_{n}\right), \nabla \widehat{\mathbf{E}}_{h}^{n+1}\right) \\
& +2 \tau \lambda\left(\nabla\left(\boldsymbol{\vartheta}^{n}+\boldsymbol{\varepsilon}_{h}^{n}\right) \odot \nabla \mathbf{b}\left(t_{n}\right), \nabla \widehat{\mathbf{E}}_{h}^{n+1}\right)+2 \tau \lambda\left(\nabla \mathbf{b}\left(t_{n}\right) \odot \nabla\left(\mathbf{b}\left(t_{n}\right)-\mathbf{b}_{h}^{n}\right), \nabla \widehat{\mathbf{E}}_{h}^{n+1}\right) \\
& +2 \tau \lambda\left(\nabla \boldsymbol{\vartheta}^{n} \odot \nabla\left(\mathbf{b}\left(t_{n}\right)-\mathbf{b}_{h}^{n}\right), \nabla \widehat{\mathbf{E}}_{h}^{n+1}\right)+2 \tau \lambda\left(\nabla \varepsilon_{h}^{n} \odot \nabla\left(\mathbf{b}\left(t_{n}\right)-\mathbf{b}_{h}^{n}\right), \nabla \widehat{\mathbf{E}}_{h}^{n+1}\right) \\
=: & \sum_{i=1} C_{i} .
\end{aligned}
$$

Then, based on the Hölder's inequality and Young inequality, we get

$$
\begin{aligned}
C_{1}+C_{2} & \leq C \tau \lambda\left(\left\|\nabla \mathbf{b}\left(t_{n+1}\right)\right\|_{L^{\infty}(\Omega)^{2}}+\left\|\nabla \mathbf{b}\left(t_{n}\right)\right\|_{L^{\infty}(\Omega)^{2}}\right)\left\|\nabla\left(\mathbf{b}\left(t_{n+1}\right)-\mathbf{b}\left(t_{n}\right)\right)\right\|_{0}\left\|\nabla \widehat{\mathbf{E}}_{h}^{n+1}\right\|_{0} \\
& \leq \frac{\tau \lambda}{64}\left\|\nabla \widehat{\mathbf{E}}_{h}^{n+1}\right\|_{0}^{2}+C \tau^{2}\left\|\mathbf{b}_{t}\right\|_{L^{2}\left(t_{n}, t_{n+1} ; H^{1}(\Omega)^{2}\right)}^{2}, \\
C_{3}+C_{4} & \leq C \tau \lambda\left\|\nabla \mathbf{b}\left(t_{n}\right)\right\|_{L^{\infty}(\Omega)^{2}}\left\|\nabla\left(\boldsymbol{\vartheta}^{n}+\varepsilon_{h}^{n}\right)\right\|_{0}\left\|\nabla \widehat{\mathbf{E}}_{h}^{n+1}\right\|_{0} \\
& \leq \frac{\tau \lambda}{64}\left\|\nabla \widehat{\mathbf{E}}_{h}^{n+1}\right\|_{0}^{2}+C \tau\left\|\nabla\left(\boldsymbol{\vartheta}^{n}+\boldsymbol{\varepsilon}_{h}^{n}\right)\right\|_{0}^{2} \leq \frac{\tau \lambda}{64}\left\|\nabla \widehat{\mathbf{E}}_{h}^{n+1}\right\|_{0}^{2}+C \tau\left(h^{2}+\left\|\nabla \boldsymbol{\varepsilon}_{h}^{n}\right\|_{0}^{2}\right), \\
C_{5} \leq C \tau & \lambda\left(\left\|\nabla \boldsymbol{\vartheta}^{n}\right\|_{L^{6}(\Omega)^{2}}\left\|\nabla \boldsymbol{\vartheta}^{n}\right\|_{L^{3}(\Omega)^{2}}+\left\|\nabla \boldsymbol{\vartheta}^{n}\right\|_{0}\left\|\nabla \boldsymbol{\varepsilon}_{h}^{n}\right\|_{L^{\infty}(\Omega)^{2}}\right)\left\|\nabla \widehat{\mathbf{E}}_{h}^{n+1}\right\|_{0} \\
& \leq \frac{\tau \lambda}{64}\left\|\nabla \widehat{\mathbf{E}}_{h}^{n+1}\right\|_{0}^{2}+C \tau\left(h^{2}+\left\|\nabla \varepsilon_{h}^{n}\right\|_{0}^{2}\right),
\end{aligned}
$$

as well as

$$
\begin{aligned}
C_{6} & \leq C \tau \lambda\left\|\nabla \varepsilon_{h}^{n}\right\|_{L^{\infty}(\Omega)^{2}}\left\|\nabla\left(\boldsymbol{\vartheta}^{n}+\varepsilon_{h}^{n}\right)\right\|_{0}\left\|\nabla \widehat{\mathbf{E}}_{h}^{n+1}\right\|_{0} \\
& \leq C \tau \lambda h^{-1}\left\|\nabla \varepsilon_{h}^{n}\right\|_{0}\left(h+\left\|\nabla \varepsilon_{h}^{n}\right\|_{0}\right)\left\|\nabla \widehat{\mathbf{E}}_{h}^{n+1}\right\|_{0} \\
& \leq \frac{\tau \lambda}{64}\left\|\nabla \widehat{\mathbf{E}}_{h}^{n+1}\right\|_{0}^{2}+C \tau\left(h^{-2}\left\|\nabla \varepsilon_{h}^{n}\right\|_{0}^{4}+\left\|\nabla \varepsilon_{h}^{n}\right\|_{0}^{2}\right),
\end{aligned}
$$

where we have applied (3.10) and the inverse inequality (2.2). Further, the bound of the last term $A_{6}$ is obtained

$$
\begin{aligned}
A_{6} \leq \frac{\mu \tau}{8} & \left(\left\|\nabla \widehat{\mathbf{E}}^{n+1}\right\|_{0}^{2}+\left\|\nabla \mathbf{F}^{n+1}\right\|_{0}^{2}\right)+C \tau^{2}\left\|\mathbf{b}_{t}(t)\right\|_{L^{2}\left(t^{n}, t^{n+1} ; H^{1}(\Omega)^{2}\right)} \\
& +C \tau\left(h^{2}+\left\|\nabla \varepsilon_{h}^{n}\right\|_{0}^{2}+h^{-2}\left\|\nabla \varepsilon_{h}^{n}\right\|_{0}^{4}\right)
\end{aligned}
$$

Finally, inserting the above terms $A_{i}$ into (3.16) leads to

$$
\begin{gathered}
\left\|\mathbf{E}^{n+1}\right\|_{0}^{2}-\left\|\mathbf{E}^{n}\right\|_{0}^{2}+\frac{1}{2}\left\|\mathbf{E}^{n+1}-\mathbf{E}^{n}\right\|_{0}^{2}+\frac{\mu \tau}{2}\left\|\nabla \widehat{\mathbf{E}}^{n+1}\right\|_{0}^{2} \\
+\left\|\nabla \rho_{h}^{n+1}\right\|_{0}^{2}+\mu \tau\left(\left\|s_{h}^{n+1}\right\|_{0}^{2}-\left\|s_{h}^{n}\right\|_{0}^{2}\right) \\
\leq C \tau\left(\left\|\nabla \mathbf{F}^{n+1}\right\|_{0}^{2}+\left\|\mathbf{E}^{n}\right\|_{0}^{2}+\left\|\mathbf{G}^{n}\right\|_{0}^{2}+h^{2}+\left\|f^{n+1}\right\|_{0}^{2}+h^{-2}\left\|\nabla \varepsilon_{h}^{n}\right\|_{0}^{4}+\left\|\nabla \varepsilon_{h}^{n}\right\|_{0}^{2}\right) \\
+C \tau^{2}+C\left\|\mathbf{F}^{n+1}\right\|_{0}^{2}+C \tau^{2}\left\|\mathbf{b}_{t}(t)\right\|_{L^{2}\left(t^{n}, t^{n+1} ; H^{1}(\Omega)^{2}\right)} .
\end{gathered}
$$


Next, sum (3.17) from $n=0$ to $N-1$ and notice $\left\|\mathbf{E}^{0}\right\|_{0}^{2}=\left(\mathbf{U}^{0}-\mathbf{u}_{h}^{0}, \mathbf{E}^{0}\right)=\left(\mathbf{U}^{0}-\mathbf{u}_{0}, \mathbf{w}_{h}\right)=0$ to yield

$$
\begin{aligned}
& \left\|\mathbf{E}^{N}\right\|_{0}^{2}+\frac{1}{2} \sum_{n=0}^{N-1}\left\|\mathbf{E}^{n+1}-\mathbf{E}^{n}\right\|_{0}^{2}+\frac{\mu \tau}{2} \sum_{n=0}^{N-1}\left\|\nabla \widehat{\mathbf{E}}^{n+1}\right\|_{0}^{2}+\sum_{n=0}^{N-1}\left\|\nabla \rho_{h}^{n+1}\right\|_{0}^{2}+\mu \tau\left\|s_{h}^{N}\right\|_{0}^{2} \\
\leq & C \tau \sum_{n=0}^{N-1}\left(\left\|\nabla \mathbf{F}^{n+1}\right\|_{0}^{2}+\left\|\mathbf{G}^{n}\right\|_{0}^{2}+\left\|f^{n+1}\right\|_{0}^{2}\right)+\tau \sum_{n=0}^{N-1}\left\|\mathbf{E}^{n}\right\|_{0}^{2} \\
& +C\left(\tau+h^{2}\right)+C \sum_{n=0}^{N-1}\left\|\mathbf{F}^{n+1}\right\|_{0}^{2}+\tau \sum_{n=0}^{N-1}\left(h^{-2}\left\|\nabla \varepsilon_{h}^{n}\right\|_{0}^{4}+\left\|\nabla \varepsilon_{h}^{n}\right\|_{0}^{2}\right) \\
\leq & C\left(\tau+h^{2}+\tau^{-1} h^{4}\right)+\tau \sum_{n=0}^{N-1}\left\|\mathbf{E}^{n}\right\|_{0}^{2}+\tau \sum_{n=0}^{N-1}\left(h^{-2}\left\|\nabla \varepsilon_{h}^{n}\right\|_{0}^{4}+\left\|\nabla \varepsilon_{h}^{n}\right\|_{0}^{2}\right)
\end{aligned}
$$

where we have used (3.9) and Lemma 3.1. On other hand, noticing $\varepsilon_{h}^{n+1}=\varepsilon^{n+1}-\boldsymbol{\vartheta}^{n+1}$, we observe that

$$
2\left(\varepsilon^{n+1}-\varepsilon^{n}, \varepsilon_{h}^{n+1}\right)=\left\|\varepsilon^{n+1}\right\|_{0}^{2}-\left\|\varepsilon^{n}\right\|_{0}^{2}+\left\|\varepsilon^{n+1}-\varepsilon^{n}\right\|_{0}^{2}-2\left(\varepsilon^{n+1}-\varepsilon^{n}, \vartheta^{n+1}\right),
$$

and

$$
\left(\nabla \varepsilon^{n+1}, \nabla \varepsilon_{h}^{n+1}\right)=\left\|\nabla \varepsilon^{n+1}\right\|_{0}^{2}-\left(\nabla \boldsymbol{\varepsilon}^{n+1}, \nabla \boldsymbol{\vartheta}^{n+1}\right)=\left\|\nabla \boldsymbol{\varepsilon}^{n+1}\right\|_{0}^{2},
$$

due to the definition of $\mathbf{b}_{h}\left(t_{n+1}\right)$. Moreover, choosing $\mathbf{r}_{h}=2 \tau \varepsilon_{h}^{n+1}$ in (3.13) gives

$$
\begin{aligned}
& \left\|\varepsilon^{n+1}\right\|_{0}^{2}-\left\|\varepsilon^{n}\right\|_{0}^{2}+\left\|\varepsilon^{n+1}-\varepsilon^{n}\right\|_{0}^{2}+2 \tau \gamma\left\|\nabla \varepsilon^{n+1}\right\|_{0}^{2} \\
= & 2\left(\varepsilon^{n+1}-\varepsilon^{n}, \vartheta^{n+1}\right)-2 \tau\left(\Pi_{h}\left(\mathbf{u}\left(t_{n+1}\right), \mathbf{b}\left(t_{n+1}\right), \varepsilon_{h}^{n+1}\right)-\Pi_{h}\left(\mathbf{u}_{h}^{n+1}, \mathbf{b}_{h}^{n+1}, \varepsilon_{h}^{n+1}\right)\right) \\
& \quad+2 \tau \gamma\left(\left|\nabla \mathbf{b}\left(t_{n+1}\right)\right|^{2} \mathbf{b}\left(t_{n+1}\right)-\left|\nabla \mathbf{b}_{h}^{n}\right|^{2} \mathbf{b}_{h}^{n}, \varepsilon_{h}^{n+1}\right)+2 \int_{t_{n}}^{t_{n+1}}\left(t-t_{n}\right)\left(\mathbf{b}_{t t}, \varepsilon_{h}^{n+1}\right) \mathrm{dt} \\
= & : \sum_{i=1}^{4} D_{i} .
\end{aligned}
$$

We now estimate each term of $A_{i}$ in (3.19) separately. Indeed, in view of the Cauchy-Schwarz and Young inequality, we deduce that

$$
\begin{aligned}
& D_{1} \leq \frac{1}{2}\left\|\varepsilon^{n+1}-\varepsilon^{n}\right\|_{0}^{2}+C\left\|\boldsymbol{\vartheta}^{n+1}\right\|_{0}^{2}, \\
& D_{4} \leq \frac{\gamma \tau}{4}\left(\left\|\nabla \varepsilon^{n+1}\right\|_{0}^{2}+\left\|\nabla \boldsymbol{\vartheta}^{n+1}\right\|_{0}^{2}\right)+C \tau^{2}\left\|\mathbf{b}_{t t}\right\|_{L^{2}\left(t^{n}, t^{n+1} ; L^{2}(\Omega)^{2}\right)}^{2}
\end{aligned}
$$

Besides, in order to estimate $A_{2}$, we add and subtract $2 \tau \Pi_{h}\left(\mathbf{u}_{h}^{n+1}, \mathbf{b}\left(t_{n+1}\right), \varepsilon_{h}^{n+1}\right)$ and bound $A_{2}$ as follows:

$$
\begin{aligned}
D_{2}=- & 2 \tau \Pi_{h}\left(\mathbf{u}\left(t_{n+1}\right)-\mathbf{u}_{h}^{n+1}, \mathbf{b}\left(t_{n+1}\right), \varepsilon_{h}^{n+1}\right)-2 \tau \Pi_{h}\left(\mathbf{u}_{h}^{n+1}, \mathbf{b}\left(t_{n+1}\right)-\mathbf{b}_{h}^{n+1}, \varepsilon_{h}^{n+1}\right) \\
=- & 2 \tau \Pi_{h}\left(\mathbf{G}^{n+1}+\mathbf{E}^{n+1}, \mathbf{b}\left(t_{n+1}\right), \varepsilon_{h}^{n+1}\right)-2 \tau \Pi_{h}\left(\mathbf{G}^{n+1}+\mathbf{E}^{n+1}, \boldsymbol{\vartheta}^{n+1}, \varepsilon_{h}^{n+1}\right) \\
& -2 \tau \Pi_{h}\left(\mathbf{u}\left(t_{n+1}\right), \boldsymbol{\vartheta}^{n+1}, \boldsymbol{\varepsilon}_{h}^{n+1}\right) \leq C \tau\left\|\mathbf{G}^{n+1}+\mathbf{E}^{n+1}\right\|_{0}\left\|\mathbf{b}\left(t_{n+1}\right)\right\|_{2}\left\|\nabla \varepsilon_{h}^{n+1}\right\|_{0} \\
& +C \tau\left\|\nabla\left(\mathbf{G}^{n+1}+\mathbf{E}^{n+1}\right)\right\|_{0}\left\|\nabla \boldsymbol{\vartheta}^{n+1}\right\|_{0}\left\|\nabla \varepsilon_{h}^{n+1}\right\|_{0}+C \tau\left\|\mathbf{u}\left(t_{n+1}\right)\right\|_{2}\left\|\boldsymbol{\vartheta}^{n+1}\right\|_{0}\left\|\nabla \varepsilon_{h}^{n+1}\right\|_{0} \\
\leq & \frac{\gamma \tau}{4}\left(\left\|\nabla \boldsymbol{\varepsilon}^{n+1}\right\|_{0}^{2}+\left\|\nabla \boldsymbol{\vartheta}^{n+1}\right\|_{0}^{2}\right)+C \tau\left(\left\|\mathbf{G}^{n+1}\right\|_{0}^{2}+\left\|\mathbf{E}^{n+1}\right\|_{0}^{2}+h^{4}\right),
\end{aligned}
$$


where we have applied (2.1) and (3.10). Further, we rewrite $D_{3}$ as

$$
\begin{aligned}
D_{3}=2 & \tau \gamma\left(\left|\nabla \mathbf{b}\left(t_{n+1}\right)\right|^{2}\left(\mathbf{b}\left(t_{n+1}\right)-\mathbf{b}\left(t_{n}\right)\right), \varepsilon_{h}^{n+1}\right)+2 \tau \gamma\left(\nabla ( \mathbf { b } ( t _ { n + 1 } ) - \mathbf { b } ( t _ { n } ) ) \cdot \nabla \left(\mathbf{b}\left(t_{n+1}\right)\right.\right. \\
& \left.\left.+\mathbf{b}\left(t_{n}\right)\right) \mathbf{b}\left(t_{n}\right), \varepsilon_{h}^{n+1}\right)+2 \tau \gamma\left(\left|\nabla \mathbf{b}\left(t_{n}\right)\right|^{2}\left(\mathbf{b}\left(t_{n}\right)-\mathbf{b}_{h}^{n}\right), \varepsilon_{h}^{n+1}\right) \\
& -4 \tau \gamma\left(\nabla\left(\mathbf{b}\left(t_{n}\right)-\mathbf{b}_{h}^{n}\right) \cdot \nabla \mathbf{b}\left(t_{n}\right)\left(\mathbf{b}\left(t_{n}\right)-\mathbf{b}_{h}^{n}\right), \varepsilon_{h}^{n+1}\right) \\
& +4 \tau \gamma\left(\nabla\left(\mathbf{b}\left(t_{n}\right)-\mathbf{b}_{h}^{n}\right) \cdot \nabla \mathbf{b}\left(t_{n}\right) \mathbf{b}\left(t_{n}\right), \varepsilon_{h}^{n+1}\right) \\
& +2 \tau \gamma\left(\left|\nabla\left(\mathbf{b}\left(t_{n}\right)-\mathbf{b}_{h}^{n}\right)\right|^{2}\left(\mathbf{b}\left(t_{n}\right)-\mathbf{b}_{h}^{n}\right), \varepsilon_{h}^{n+1}\right)-2 \tau \gamma\left(\left|\nabla\left(\mathbf{b}\left(t_{n}\right)-\mathbf{b}_{h}^{n}\right)\right|^{2} \mathbf{b}\left(t_{n}\right), \varepsilon_{h}^{n+1}\right) \\
=: & \sum_{i=1}^{7} F_{i} .
\end{aligned}
$$

We now estimate $F_{i}$ in the above equation separately. According to the Cauchy-Schwarz and Young inequality, the inverse inequality (2.2) and (3.10), we have

$$
\begin{aligned}
F_{1} & \leq \frac{\gamma \tau}{56}\left\|\nabla \varepsilon_{h}^{n+1}\right\|_{0}^{2}+C \tau^{2}\left\|\nabla \mathbf{b}\left(t_{n+1}\right)\right\|_{L^{\infty}(\Omega)^{2}}^{4}\left\|\mathbf{b}_{t}\right\|_{L^{2}\left(t_{n}, t_{n+1} ; L^{2}(\Omega)^{2}\right)}^{2}, \\
F_{2} & \leq \frac{\gamma \tau}{56}\left\|\nabla \varepsilon_{h}^{n+1}\right\|_{0}^{2}+C \tau^{2}\left\|\nabla\left(\mathbf{b}\left(t_{n+1}\right)+\mathbf{b}\left(t_{n}\right)\right)\right\|_{L^{\infty}(\Omega)^{2}}^{2}\left\|\mathbf{b}\left(t_{n}\right)\right\|_{L^{\infty}(\Omega)^{2}}^{2}\left\|\mathbf{b}_{t}\right\|_{L^{2}\left(t_{n}, t_{n+1} ; L^{2}(\Omega)^{2}\right)}^{2}, \\
F_{3} & \leq \frac{\gamma \tau}{56}\left\|\nabla \varepsilon_{h}^{n+1}\right\|_{0}^{2}+C \tau\left\|\nabla \mathbf{b}\left(t_{n}\right)\right\|_{L^{\infty}(\Omega)^{2}}^{4}\left(h^{4}+\left\|\varepsilon_{h}^{n}\right\|_{0}^{2}\right), \\
F_{4} & \leq \frac{\gamma \tau}{56}\left\|\nabla \varepsilon_{h}^{n+1}\right\|_{0}^{2}+C \tau\left\|\nabla\left(\mathbf{b}\left(t_{n}\right)-\mathbf{b}_{h}^{n}\right)\right\|_{0}^{2}\left\|\nabla \mathbf{b}\left(t_{n}\right)\right\|_{L^{\infty}(\Omega)^{2}}^{2}\left\|\mathbf{b}\left(t_{n}\right)-\mathbf{b}_{h}^{n}\right\|_{L^{\infty}(\Omega)^{2}}^{2} \\
& \leq \frac{\gamma \tau}{56}\left\|\nabla \varepsilon_{h}^{n+1}\right\|_{0}^{2}+C \tau\left(h^{4}+\left\|\nabla \varepsilon_{h}^{n}\right\|_{0}^{4}\right), \\
F_{5} & \leq \frac{\gamma \tau}{56}\left\|\nabla \varepsilon_{h}^{n+1}\right\|_{0}^{2}+C \tau\left\|\nabla \mathbf{b}\left(t_{n}\right)\right\|_{L^{\infty}(\Omega)^{2}}^{2}\left\|\mathbf{b}\left(t_{n}\right)\right\|_{L^{\infty}(\Omega)^{2}}^{2}\left(h^{2}+\left\|\nabla \varepsilon_{h}^{n}\right\|_{0}^{2}\right), \\
F_{6} & \leq \frac{\gamma \tau}{56}\left\|\nabla \varepsilon_{h}^{n+1}\right\|_{0}^{2}+C \tau\left\|\mathbf{b}\left(t_{n}\right)-\mathbf{b}_{h}^{n}\right\|_{L^{\infty}(\Omega)^{2}}^{2}\left\|\nabla\left(\mathbf{b}\left(t_{n}\right)-\mathbf{b}_{h}^{n}\right)\right\|_{L^{3}(\Omega)^{2}}^{2}\left\|\nabla\left(\mathbf{b}\left(t_{n}\right)-\mathbf{b}_{h}^{n}\right)\right\|_{L^{6}(\Omega)^{2}}^{2} \\
& \leq \frac{\gamma \tau}{56}\left\|\nabla \varepsilon_{h}^{n+1}\right\|_{0}^{2}+C \tau\left(h^{4}+h^{-2}\left\|\nabla \varepsilon_{h}^{n}\right\|_{0}^{6}\right), \\
F_{7} & \leq C \gamma \tau\left\|\nabla\left(\mathbf{b}\left(t_{n}\right)-\mathbf{b}_{h}^{n}\right)\right\|_{L^{6}(\Omega)^{2}}\left\|\nabla\left(\mathbf{b}\left(t_{n}\right)-\mathbf{b}_{h}^{n}\right)\right\|_{L^{3}(\Omega)^{2}}\left\|\mathbf{b}\left(t_{n}\right)\right\|_{L^{\infty}(\Omega)^{2}}\left\|\varepsilon_{h}^{n+1}\right\|_{0} \\
& \leq C \gamma \tau h^{-1}\left\|\nabla\left(\mathbf{b}\left(t_{n}\right)-\mathbf{b}_{h}^{n}\right)\right\|_{0}^{2}\left\|\varepsilon_{h}^{n+1}\right\|_{0} \\
& \leq \frac{\gamma \tau}{56}\left\|\nabla \varepsilon_{h}^{n+1}\right\|_{0}^{2}+C \tau\left(h^{2}+h^{-2}\left\|\nabla \varepsilon_{h}^{n}\right\|_{0}^{4}\right) .
\end{aligned}
$$

Hence, we get the bound of $D_{3}$

$$
\begin{aligned}
D_{3} \leq \frac{\gamma \tau}{4}\left(\left\|\nabla \varepsilon^{n+1}\right\|_{0}^{2}+\left\|\nabla \boldsymbol{\vartheta}^{n+1}\right\|_{0}^{2}\right)+C \tau^{2}\left\|\mathbf{b}_{t}\right\|_{L^{2}\left(t_{n}, t_{n+1} ; L^{2}(\Omega)^{2}\right)}^{2} \\
+C \tau\left(h^{2}+\left\|\nabla \varepsilon_{h}^{n}\right\|_{0}^{2}+h^{-2}\left\|\nabla \varepsilon_{h}^{n}\right\|_{0}^{4}+h^{-2}\left\|\nabla \varepsilon_{h}^{n}\right\|_{0}^{6}\right) .
\end{aligned}
$$

Finally, plugging the above bounds of $D_{i}$ into (3.19), we have

$$
\begin{aligned}
& \left\|\varepsilon^{n+1}\right\|_{0}^{2}-\left\|\varepsilon^{n}\right\|_{0}^{2}+\frac{1}{2}\left\|\varepsilon^{n+1}-\varepsilon^{n}\right\|_{0}^{2}+\tau \gamma\left\|\nabla \varepsilon^{n+1}\right\|_{0}^{2} \\
& \leq C h^{4}+C \tau^{2}\left(\left\|\mathbf{b}_{t t}\right\|_{L^{2}\left(t^{n}, t^{n+1} ; L^{2}(\Omega)^{2}\right)}^{2}+\left\|\mathbf{b}_{t}\right\|_{L^{2}\left(t_{n}, t_{n+1} ; L^{2}(\Omega)^{2}\right)}^{2}\right) \\
& \quad+C \tau\left(\left\|\mathbf{G}^{n+1}\right\|_{0}^{2}+\left\|\mathbf{E}^{n+1}\right\|_{0}^{2}+h^{2}+\left\|\nabla \varepsilon_{h}^{n}\right\|_{0}^{2}+h^{-2}\left\|\nabla \varepsilon_{h}^{n}\right\|_{0}^{4}+h^{-2}\left\|\nabla \varepsilon_{h}^{n}\right\|_{0}^{6}\right) .
\end{aligned}
$$


Next, sum (3.20) from $n=0$ to $N-1$ and notice $\left\|\varepsilon^{0}\right\|_{0}^{2}=\left(\mathbf{b}_{0}-\mathbf{b}_{h}^{0}, \varepsilon^{0}\right)=0$ to yield

$$
\begin{aligned}
& \left\|\varepsilon^{N}\right\|_{0}^{2}+\frac{1}{2} \sum_{n=0}^{N-1}\left\|\varepsilon^{n+1}-\varepsilon^{n}\right\|_{0}^{2}+\tau \gamma \sum_{n=0}^{N-1}\left\|\nabla \varepsilon^{n+1}\right\|_{0}^{2} \\
& \leq C\left(\tau^{-1} h^{4}+\tau^{2}+h^{2}\right)+C \tau \sum_{n=0}^{N-1}\left\|\mathbf{E}^{n+1}\right\|_{0}^{2} \\
& \quad+\tau \sum_{n=0}^{N-1}\left(\left\|\nabla \varepsilon_{h}^{n}\right\|_{0}^{2}+h^{-2}\left\|\nabla \varepsilon_{h}^{n}\right\|_{0}^{4}+h^{-2}\left\|\nabla \varepsilon_{h}^{n}\right\|_{0}^{6}\right),
\end{aligned}
$$

where we have applied Lemma 3.1. Then, adding (3.21) and (3.18) to get

$$
\begin{gathered}
\left\|\mathbf{E}^{N}\right\|_{0}^{2}+\frac{1}{2} \sum_{n=0}^{N-1}\left\|\mathbf{E}^{n+1}-\mathbf{E}^{n}\right\|_{0}^{2}+\frac{\mu \tau}{2} \sum_{n=0}^{N-1}\left\|\nabla \widehat{\mathbf{E}}^{n+1}\right\|_{0}^{2}+\sum_{n=0}^{N-1}\left\|\nabla \rho_{h}^{n+1}\right\|_{0}^{2} \\
+\mu \tau\left\|s_{h}^{N}\right\|_{0}^{2}+\left\|\varepsilon^{N}\right\|_{0}^{2}+\frac{1}{2} \sum_{n=0}^{N-1}\left\|\varepsilon^{n+1}-\varepsilon^{n}\right\|_{0}^{2}+\tau \gamma \sum_{n=0}^{N-1}\left\|\nabla \varepsilon^{n+1}\right\|_{0}^{2} \\
\leq C\left(\tau+h^{2}+\tau^{-1} h^{4}\right)+\tau \sum_{n=0}^{N-1}\left\|\mathbf{E}^{n+1}\right\|_{0}^{2} \\
+\tau \sum_{n=0}^{N-1}\left(h^{-2}\left\|\nabla \varepsilon_{h}^{n}\right\|_{0}^{4}+\left\|\nabla \varepsilon_{h}^{n}\right\|_{0}^{2}+h^{-2}\left\|\nabla \varepsilon_{h}^{n}\right\|_{0}^{6}\right) .
\end{gathered}
$$

In what follows, in order to derive final estimate for error, we need establish bound of $\left\|\nabla \varepsilon_{h}^{n}\right\|_{0}^{2}$. First, setting $\mathbf{r}_{h}=\tau^{-1}\left(\varepsilon_{h}^{n+1}-\varepsilon_{h}^{n}\right)$ in (3.13) yields

$$
\begin{aligned}
& \tau^{-2}\left\|\varepsilon_{h}^{n+1}-\varepsilon_{h}^{n}\right\|_{0}^{2}+\frac{\tau^{-1} \gamma}{2}\left(\left\|\nabla \varepsilon_{h}^{n+1}\right\|_{0}^{2}-\left\|\nabla \varepsilon_{h}^{n}\right\|_{0}^{2}+\left\|\nabla\left(\varepsilon_{h}^{n+1}-\varepsilon_{h}^{n}\right)\right\|_{0}^{2}\right) \\
=-\tau^{-2}\left(\boldsymbol{\vartheta}^{n+1}-\boldsymbol{\vartheta}^{n}, \varepsilon_{h}^{n+1}-\varepsilon_{h}^{n}\right)-\tau^{-1}\left(\Pi_{h}\left(\mathbf{u}\left(t_{n+1}\right), \mathbf{b}\left(t_{n+1}\right), \varepsilon_{h}^{n+1}-\varepsilon_{h}^{n}\right)\right. & \\
& \left.-\Pi_{h}\left(\mathbf{u}_{h}^{n+1}, \mathbf{b}_{h}^{n+1}, \varepsilon_{h}^{n+1}-\varepsilon_{h}^{n}\right)\right)+\tau^{-1} \gamma\left(\left|\nabla \mathbf{b}\left(t_{n+1}\right)\right|^{2} \mathbf{b}\left(t_{n+1}\right)\right. \\
& \left.-\left|\nabla \mathbf{b}_{h}^{n}\right|^{2} \mathbf{b}_{h}^{n}, \varepsilon_{h}^{n+1}-\varepsilon_{h}^{n}\right)+\tau^{-2} \int_{t_{n}}^{t_{n+1}}\left(t-t_{n}\right)\left(\mathbf{b}_{t t}, \varepsilon_{h}^{n+1}-\varepsilon_{h}^{n}\right) \mathrm{dt} \\
=: & \sum_{i=1}^{4} G_{i} .
\end{aligned}
$$

Then, we estimate these $G_{i}$ separately. Making use of the Cauchy-Schwarz and Young inequality, we observe that

$$
\begin{aligned}
G_{1} & \leq \frac{\tau^{-2}}{4}\left\|\varepsilon_{h}^{n+1}-\varepsilon_{h}^{n}\right\|_{0}^{2}+C \tau^{-2}\left\|\boldsymbol{\vartheta}^{n+1}-\boldsymbol{\vartheta}^{n}\right\|_{0}^{2} \\
& \leq \frac{\tau^{-2}}{4}\left\|\varepsilon_{h}^{n+1}-\varepsilon_{h}^{n}\right\|_{0}^{2}+C \tau^{-1}\left\|\boldsymbol{\vartheta}_{t}\right\|_{L^{2}\left(t^{n}, t^{n+1} ; L^{2}(\Omega)^{2}\right)}^{2},
\end{aligned}
$$

as well as

$$
G_{4} \leq \frac{\gamma \tau^{-1}}{8}\left\|\nabla\left(\varepsilon_{h}^{n+1}-\varepsilon_{h}^{n}\right)\right\|_{0}^{2}+C\left\|\mathbf{b}_{t t}\right\|_{L^{2}\left(t^{n}, t^{n+1} ; L^{2}(\Omega)^{2}\right)}^{2} .
$$


In addition, by employing (2.1), we obtain

$$
\begin{aligned}
G_{2}= & -\tau^{-1} \Pi_{h}\left(\mathbf{G}^{n+1}+\mathbf{E}^{n+1}, \mathbf{b}\left(t_{n+1}\right), \boldsymbol{\varepsilon}_{h}^{n+1}-\boldsymbol{\varepsilon}_{h}^{n}\right) \\
& +\tau^{-1} \Pi_{h}\left(\mathbf{G}^{n+1}+\mathbf{E}^{n+1}, \boldsymbol{\vartheta}^{n+1}+\boldsymbol{\varepsilon}_{h}^{n}, \boldsymbol{\varepsilon}_{h}^{n+1}-\boldsymbol{\varepsilon}_{h}^{n}\right) \\
& \quad-\tau^{-1} \Pi_{h}\left(\mathbf{u}\left(t_{n+1}\right), \boldsymbol{\vartheta}^{n+1}+\boldsymbol{\varepsilon}_{h}^{n}, \boldsymbol{\varepsilon}_{h}^{n+1}-\boldsymbol{\varepsilon}_{h}^{n}\right) \\
\leq & C \tau^{-1}\left\|\nabla\left(\mathbf{G}^{n+1}+\mathbf{E}^{n+1}\right)\right\|_{0}\left\|\mathbf{b}\left(t_{n+1}\right)\right\|_{2}\left\|\varepsilon_{h}^{n+1}-\varepsilon_{h}^{n}\right\|_{0} \\
& \quad+C \tau^{-1}\left\|\nabla\left(\mathbf{G}^{n+1}+\mathbf{E}^{n+1}\right)\right\| \nabla\left(\boldsymbol{\vartheta}^{n+1}+\boldsymbol{\varepsilon}_{h}^{n}\right)\left\|_{0}\right\| \nabla\left(\varepsilon_{h}^{n+1}-\boldsymbol{\varepsilon}_{h}^{n}\right) \|_{0} \\
& \quad+C \tau^{-1}\left\|\mathbf{u}\left(t_{n+1}\right)\right\|_{2}\left\|\nabla\left(\boldsymbol{\vartheta}^{n+1}+\varepsilon_{h}^{n}\right)\right\|\left\|_{0}^{n+1}-\varepsilon_{h}^{n}\right\|_{0} \\
\leq & \frac{\tau^{-2}}{4}\left\|\varepsilon^{n+1}-\varepsilon^{n}\right\|_{0}^{2}+C\left(\left\|\nabla \boldsymbol{\vartheta}^{n+1}\right\|_{0}^{2}+\left\|\nabla \varepsilon_{h}^{n}\right\|_{0}^{2}+\left\|\nabla \mathbf{G}^{n+1}\right\|_{0}^{2}+\left\|\nabla \mathbf{E}^{n+1}\right\|_{0}^{2}\right) \\
& \quad+\frac{\gamma \tau^{-1}}{8}\left\|\nabla\left(\varepsilon_{h}^{n+1}-\varepsilon_{h}^{n}\right)\right\|_{0}^{2}+C \tau^{-1}\left(\left\|\nabla \mathbf{G}^{n+1}\right\|_{0}^{2}+\left\|\nabla \mathbf{E}^{n+1}\right\|_{0}^{2}\right)\left(\left\|\nabla \boldsymbol{\vartheta}^{n+1}\right\|_{0}^{2}+\left\|\nabla \varepsilon_{h}^{n}\right\|_{0}^{2}\right) .
\end{aligned}
$$

Arguing in exactly the same way as $G_{3}$ in (3.19), we get

$$
\begin{aligned}
G_{3} \leq & \frac{\tau^{-2}}{4}\left\|\varepsilon^{n+1}-\varepsilon^{n}\right\|_{0}^{2}+C \tau\left\|\mathbf{b}_{t}\right\|_{L^{2}\left(t_{n}, t_{n+1} ; L^{2}(\Omega)^{2}\right)}^{2} \\
& +C\left(h^{2}+\left\|\nabla \varepsilon_{h}^{n}\right\|_{0}^{2}+h^{-2}\left\|\nabla \varepsilon_{h}^{n}\right\|_{0}^{4}+h^{-2}\left\|\nabla \varepsilon_{h}^{n}\right\|_{0}^{6}\right) .
\end{aligned}
$$

Plug the above bounds of $A_{i}$ into (3.23) and reorganize the ensuing equation. Then,

$$
\begin{aligned}
& \frac{\tau^{-2}}{4}\left\|\varepsilon_{h}^{n+1}-\varepsilon_{h}^{n}\right\|_{0}^{2}+\frac{\tau^{-1} \gamma}{2}\left(\left\|\nabla \varepsilon_{h}^{n+1}\right\|_{0}^{2}-\left\|\nabla \varepsilon_{h}^{n}\right\|_{0}^{2}\right)+\frac{\tau^{-1} \gamma}{4}\left\|\nabla\left(\varepsilon_{h}^{n+1}-\varepsilon_{h}^{n}\right)\right\|_{0}^{2} \\
& \leq C \tau^{-1}\left\|\boldsymbol{\vartheta}_{t}\right\|_{L^{2}\left(t^{n}, t^{n+1} ; L^{2}(\Omega)^{2}\right)}^{2}+C\left\|\mathbf{b}_{t t}\right\|_{L^{2}\left(t^{n}, t^{n+1} ; L^{2}(\Omega)^{2}\right)}^{2}+C\left(\left\|\nabla \mathbf{G}^{n+1}\right\|_{0}^{2}+\left\|\nabla \mathbf{E}^{n+1}\right\|_{0}^{2}\right) \\
& \quad+C \tau^{-1}\left(\left\|\nabla \mathbf{G}^{n+1}\right\|_{0}^{2}+\left\|\nabla \mathbf{E}^{n+1}\right\|_{0}^{2}\right)\left(\left\|\nabla \boldsymbol{\vartheta}^{n+1}\right\|_{0}^{2}+\left\|\nabla \varepsilon_{h}^{n}\right\|_{0}^{2}\right) \\
& \quad+C\left(\left\|\nabla \boldsymbol{\vartheta}^{n+1}\right\|_{0}^{2}+\left\|\nabla \varepsilon_{h}^{n}\right\|_{0}^{2}\right)+C \tau\left\|\mathbf{b}_{t}\right\|_{L^{2}\left(t_{n}, t_{n+1} ; L^{2}(\Omega)^{2}\right)}^{2} \\
& \quad+C\left(h^{2}+\left\|\nabla \varepsilon_{h}^{n}\right\|_{0}^{2}+h^{-2}\left\|\nabla \varepsilon_{h}^{n}\right\|_{0}^{4}+h^{-2}\left\|\nabla \varepsilon_{h}^{n}\right\|_{0}^{6}\right) .
\end{aligned}
$$

Second, multiply (3.24) by $\tau^{2}$ and sum the resulting inequality from $n=0$ to $N-1$ to get

$$
\begin{gathered}
\frac{1}{4} \sum_{n=0}^{N-1}\left\|\varepsilon_{h}^{n+1}-\varepsilon_{h}^{n}\right\|_{0}^{2}+\frac{\tau \gamma}{2}\left\|\nabla \varepsilon_{h}^{N}\right\|_{0}^{2}+\frac{\tau \gamma}{4} \sum_{n=0}^{N-1}\left\|\nabla\left(\varepsilon_{h}^{n+1}-\varepsilon_{h}^{n}\right)\right\|_{0}^{2} \\
\leq C \tau^{2}+C \tau^{2} \sum_{n=0}^{N-1}\left\|\nabla \mathbf{E}^{n+1}\right\|_{0}^{2}+C \tau \sum_{n=0}^{N-1}\left(\left\|\nabla \mathbf{G}^{n+1}\right\|_{0}^{2}+\left\|\nabla \mathbf{E}^{n+1}\right\|_{0}^{2}\right)\left(h^{2}+\left\|\nabla \varepsilon_{h}^{n}\right\|_{0}^{2}\right) \\
+C \tau\left(h^{2}+\left\|\nabla \varepsilon_{h}^{n}\right\|_{0}^{2}+h^{-2}\left\|\nabla \varepsilon_{h}^{n}\right\|_{0}^{4}+h^{-2}\left\|\nabla \varepsilon_{h}^{n}\right\|_{0}^{6}\right) .
\end{gathered}
$$

Now we prove that $\left\|\nabla \varepsilon_{h}^{n}\right\|_{0}^{2} \leq \tau$ for $0 \leq n \leq N$ by using mathematical induction method. Clearly, this inequality holds for $n=0$. If we assume that this inequality holds for $n \leq N-1$, then the inequality $(3.25)$ and $(3.22)$ reduces to

$$
\begin{gathered}
\frac{\tau \gamma}{2}\left\|\nabla \varepsilon_{h}^{N}\right\|_{0}^{2} \leq C \tau^{2}+C \tau^{2} \sum_{n=0}^{N-1}\left\|\nabla \mathbf{E}^{n+1}\right\|_{0}^{2}+C \tau\left(h^{2}+\tau\right) \sum_{n=0}^{N-1}\left(\left\|\nabla \mathbf{G}^{n+1}\right\|_{0}^{2}+\left\|\nabla \mathbf{E}^{n+1}\right\|_{0}^{2}\right) \\
+C \tau\left(h^{2}+\tau+\tau^{2} h^{-2}+\tau^{3} h^{-2}\right) \\
\leq C\left(\tau h^{2}+h^{-2} \tau^{3}+\tau^{2}+\tau^{4} h^{-2}\right)+C \tau\left(h^{2}+\tau\right) \sum_{n=0}^{N-1}\left\|\nabla \mathbf{E}^{n+1}\right\|_{0}^{2}
\end{gathered}
$$


and

$$
\begin{aligned}
& \left\|\mathbf{E}^{N}\right\|_{0}^{2}+\frac{1}{2} \sum_{n=0}^{N-1}\left\|\mathbf{E}^{n+1}-\mathbf{E}^{n}\right\|_{0}^{2}+\frac{\mu \tau}{2} \sum_{n=0}^{N-1}\left\|\nabla \widehat{\mathbf{E}}^{n+1}\right\|_{0}^{2}+\sum_{n=0}^{N-1}\left\|\nabla \rho_{h}^{n+1}\right\|_{0}^{2}+\mu \tau\left\|s_{h}^{N}\right\|_{0}^{2} \\
& +\left\|\varepsilon^{N}\right\|_{0}^{2}+\frac{1}{2} \sum_{n=0}^{N-1}\left\|\varepsilon^{n+1}-\varepsilon^{n}\right\|_{0}^{2}+\tau \gamma \sum_{n=0}^{N-1}\left\|\nabla \varepsilon^{n+1}\right\|_{0}^{2} \\
& \leq C\left(\tau+h^{2}+\tau^{-1} h^{4}+h^{-2} \tau^{3}+h^{-2} \tau^{2}\right)+\tau \sum_{n=0}^{N-1}\left\|\mathbf{E}^{n+1}\right\|_{0}^{2}
\end{aligned}
$$

respectively. Hence, the Grönwall lemma in combination with (3.27) yields

$$
\begin{aligned}
& \left\|\mathbf{E}^{N}\right\|_{0}^{2}+\frac{1}{2} \sum_{n=0}^{N-1}\left\|\mathbf{E}^{n+1}-\mathbf{E}^{n}\right\|_{0}^{2}+\frac{\mu \tau}{2} \sum_{n=0}^{N-1}\left\|\nabla \widehat{\mathbf{E}}^{n+1}\right\|_{0}^{2}+\sum_{n=0}^{N-1}\left\|\nabla \rho_{h}^{n+1}\right\|_{0}^{2} \\
& \quad+\mu \tau\left\|s_{h}^{N}\right\|_{0}^{2}+\left\|\varepsilon^{N}\right\|_{0}^{2}+\frac{1}{2} \sum_{n=0}^{N-1}\left\|\varepsilon^{n+1}-\varepsilon^{n}\right\|_{0}^{2}+\tau \gamma \sum_{n=0}^{N-1}\left\|\nabla \varepsilon^{n+1}\right\|_{0}^{2} \\
& \leq C\left(\tau+h^{2}+\tau^{-1} h^{4}+h^{-2} \tau^{3}+h^{-2} \tau^{2}\right),
\end{aligned}
$$

which implies that

$$
\tau \sum_{n=0}^{N-1}\left\|\nabla \mathbf{E}^{n+1}\right\|_{0}^{2} \leq \tau \sum_{n=0}^{N-1}\left\|\nabla \widehat{\mathbf{E}}^{n+1}\right\|_{0}^{2} \leq C\left(\tau+h^{2}+\tau^{-1} h^{4}+h^{-2} \tau^{3}+h^{-2} \tau^{2}\right)
$$

Inserting (3.29) into (3.26), we completes the induction if $\tau=\mathcal{O}\left(h^{2}\right)$ is valid.

Finally, in right of (3.28), we get

$$
\begin{aligned}
& \left\|\mathbf{E}^{N}\right\|_{0}^{2}+\frac{1}{2} \sum_{n=0}^{N-1}\left\|\mathbf{E}^{n+1}-\mathbf{E}^{n}\right\|_{0}^{2}+\frac{\mu \tau}{2} \sum_{n=0}^{N-1}\left\|\nabla \mathbf{E}^{n+1}\right\|_{0}^{2}+\sum_{n=0}^{N-1}\left\|\nabla \rho_{h}^{n+1}\right\|_{0}^{2} \\
& \quad+\mu \tau\left\|s_{h}^{N}\right\|_{0}^{2}+\left\|\varepsilon^{N}\right\|_{0}^{2}+\frac{1}{2} \sum_{n=0}^{N-1}\left\|\varepsilon^{n+1}-\varepsilon^{n}\right\|_{0}^{2}+\tau \gamma \sum_{n=0}^{N-1}\left\|\nabla \varepsilon^{n+1}\right\|_{0}^{2} \\
& \leq C\left(\tau+h^{2}\right) .
\end{aligned}
$$

Hence, from (3.30) and the triangle inequality, we finish the proof.

\section{Numerical Tests}

In this section, we assess numerical performance of the finite element algorithm based on the gauge-Uzawa method for the nematic liquid crystal flow. It will be checked by a known analytical solution problem. The main goal of the experiment is to verify convergence rates of Algorithm 2.1, which is shown in Theorem 3.1. Denote errors by

$$
\operatorname{Err}(\mathbf{u})=\left(\tau \sum_{n=0}^{N-1}\left\|\nabla\left(\mathbf{u}\left(t_{n+1}\right)-\mathbf{u}_{h}^{n+1}\right)\right\|_{0}^{2}\right)^{1 / 2}, \quad \operatorname{Err}(\mathbf{b})=\left(\tau \sum_{n=0}^{N-1}\left\|\nabla\left(\mathbf{b}\left(t_{n+1}\right)-\mathbf{b}_{h}^{n+1}\right)\right\|_{0}^{2}\right)^{1 / 2} .
$$


Consider the nematic liquid crystal model with parameters $\lambda=1, \mu=1$ and $\gamma=1$ in the unit circle $\Omega=\left\{(x, y): x^{2}+y^{2}<1\right\}$. Besides, the initial data are taken as

$$
\mathbf{u}_{0}=\mathbf{0}, \quad \mathbf{b}_{0}=(\sin (a), \cos (a))
$$

with $a=\pi\left(x^{2}+y^{2}\right)^{2}$. The body force on the flow is chosen $\mathbf{f}=\mathbf{0}$ and the final time $T=0.1$.

Table 4.1: Numerical errors and convergence rates.

\begin{tabular}{|llccc|}
\hline $1 / h$ & $\operatorname{Err}(\mathbf{b})$ & rate & $\operatorname{Err}(\mathbf{u})$ & rate \\
\hline 20 & $4.949 \mathrm{E}-1$ & - & $1.693 \mathrm{E}-1$ & - \\
40 & $1.482 \mathrm{E}-1$ & 1.7 & $8.201 \mathrm{E}-2$ & 1.0 \\
60 & $1.041 \mathrm{E}-1$ & 0.9 & $5.698 \mathrm{E}-2$ & 0.9 \\
80 & $8.399 \mathrm{E}-2$ & 0.8 & $4.385 \mathrm{E}-2$ & 0.9 \\
\hline
\end{tabular}

The exact solution to this problem is unknown. Thus, we take the numerical solution by the standard Galerkin method element computed on a very fine mesh $h=1 / 150$ as the "exact" solution for the purpose of comparison. Here, we choose the values of $h$, i.e., $1 / 20,1 / 40,1 / 60$ and $1 / 80$ with the time step $\tau=h^{2}$. We display the convergence orders and errors of the presented method in Table 4.1. From this table, we can see that the presented method works well and keeps the convergence rates just like the theoretical analysis.

Acknowledgments. This work is supported by the Natural Science Foundation of China (grant number 11861067). The authors would like to thank the editor and anonymous referees for their helpful comments and suggestions which lead to a considerably improved presentation.

\section{References}

[1] R. An and J. Su, Optimal error estimates of semi-implicit Galerkin method for time-dependent nematic liquid crystal flows, J. Sci. Comput., 74 (2018), 979-1008.

[2] S. Badia, F. Guillén-Gónzalez and J. V. Gutiérrez-Santacreu, An overview on numerical analyses of nematic liquid crystal flows, Arch. Comput. Methods Eng., 18 (2011), 285-313.

[3] X. Bao, R. Chen and H. Zhang, Constraint-preserving energy-stable scheme for the 2D simplified Ericksen-Leslie system, J. Comput. Math., (2020), doi:10.4208/jcm.1906-m2018-0144.

[4] R. Becker, X.B. Feng and A. Prohl, Finite element approximations of the Ericksen-Leslie model for nematic liquid crystal flow, SIAM J. Numer. Anal., 46 (2008), 1704-1731.

[5] S. Brenner and L. Scott, The Mathematical Theory of Finite Element Methods, Springer, Berlin, 1994.

[6] R.C. Cabrales, F. Guillén-Gónzalez and J. V. Gutiérrez-Santacreu, A time-splitting finite-element stable approximation for the Ericksen-Leslie equations, SIAM J. Sci. Comput., 37 (2015), B261B282.

[7] R.C. Cabrales, F. Guillén-Gónzalez and J. V. Gutiérrez-Santacreu, A projection-based timesplitting algorithm for approximating nematic liquid crystal flows with stretching, ZAMM: Z. Angew. Math. Mech., 97 (2017), 1204-1219.

[8] Q. Du, B. Guo and J. Shen, Fourier spectral approximation to a dissipative system modeling the flow of liquid crystals, SIAM J. Numer. Anal., 39 (2001), 735-762.

[9] J. Ericksen, Conservation laws for liquid crystals, Trans. Soc. Rheol., 5 (1961), 22-34.

[10] J. Ericksen, Continuum theory of nematic liquid crystals, Res. Mech., 21 (1987), 381-392. 
[11] V. Girault and F. Guillén-Gónzalez, Mixed formulation, approximation and decoupling algorithm for a penalized nematic liquid crystals model, Math. Comp., 80: (2011), 781-819.

[12] F. Guillén-Gónzalez and J. V. Gutiérrez-Santacreu, A linear mixed finite element scheme for a nematic Ericksen-Leslie liquid crystal model, ESAIM: Math. Model. Numer. Anal., 47 (2013), 1433-1464.

[13] F. Guillén-Gónzalez and J. Koko, A splitting in time scheme and augmented lagrangian method for a nematic liquid crystal problem, J. Sci. Comput., 65 (2015), 1129-1144.

[14] M. Hong, Global existence of solutions of the simplified Ericksen-Leslie system in dimension two, Calc. Var. Part. Differ. Equ., 40 (2011), 15-36.

[15] T. Huang and C. Wang, Blow up criterion for nematic liquid crystal flows, Commun. Part. Differ. Equ., 37 (2012), 875-884.

[16] F. Leslie, Some constitutive equations for liquid crystals, Arch. Ration. Mech. Anal., 28 (1968), 265-283.

[17] B. Y. Li, W.W. Sun, Linearized fe approximations to a nonlinear gradient flow, SIAM J. Numer. Anal., 52 (2014), 2623-2646.

[18] F.H. Lin, Nonlinear theory of defects in nematics liquid crystals: Phase transitation and flow phenomena, Commun. Pure Appl. Math., 42 (1989), 789-814.

[19] F.H. Lin, J. Lin and C. Wang, Liquid crystal flows in two dimensions, Arch. Ration. Mech. Anal., 197 (2010), 297-336.

[20] F.H. Lin and C. Liu, Existence of solutions for the Ericksen-Leslie system, Arch. Ration. Mech. Anal., 154 (2000), 135-156.

[21] C. Liu and N.J. Walkington, Approximation of liquid crystal flows, SIAM J. Numer. Anal., 37 (2000), 725-741.

[22] C. Liu and N. Walkington, Mixed method for the approximation of liquid crystal flow, ESAIM: Math. Model. Numer. Anal., 36 (2002), 205-222.

[23] F.H. Lin and C. Wang, On the uniqueness of heat flow of harmonic maps and hydrodynamic flow of nematic liquid crystals, Chin. Ann. Math. Ser. B, 31 (2010), 921-938.

[24] R. Nochetto and J.H. Pyo, A finite element gauge-Uzawa method. Part I: The Navier-Stokes equations, SIAM J. Numer. Anal., 43 (2005), 1043-1068.

[25] R. Nochetto and J.H. Pyo, The gauge-Uzawa finite element method Part II: The Boussinesq equations, Math. Models Meth. Appl. Sci., 10 (2006), 1599-1626.

[26] J. Pyo, Optimal error estimate for semi-discrete gauge-Uzawa method for the Navier-Stokes equations, Bull. Korean Math. Soc., 46 (2009), 627-644.

[27] J. Pyo, Error estimate for the second order semi-discrete stabilized gauge-Uzawa method for the Navier-Stokes equations, Int. J. Numer. Anal. Model., 10 (2013), 24-41.

[28] J. Pyo, Fully discrete finite element approximation for the stabilized gauge-Uzawa method to solve the Boussinesq equations, J. Appl. Math., 2013 (2013), 1-21.

[29] J. Pyo and J. Shen, Gauge-Uzawa methods for incompressible flows with variable density, $J$. Comput. Phys., 221 (2007), 181-197.

[30] Z. Si, W. Li and Y. Wang, A gauge-Uzawa finite element method for the time-dependent Viscoelastic Oldroyd flows, J. Math. Anal. Appl., 425 (2015), 96-110.

[31] Z. Si, X. Song and P.Z. Huang, Modified characteristics gauge-Uzawa finite element method for time dependent conduction-convection problems, J. Sci. Comput., 58 (2014), 1-24.

[32] C. Wang, Well-posedness for the heat flow of harmonic maps and the liquid crystal flow with rough initial data, Arch. Ration. Mech. Anal., 200 (2011), 1-19.

[33] J. Wu, J. Shen and X. Feng, Unconditionally stable Gauge-Uzawa finite element schemes for incompressible natural convection problems with variable density, J. Comput. Phys., 348 (2017), 776-789.

[34] X. Xu and Z. Zhang, Global regularity and uniqueness of weak solution for the 2D liquid crystal flows, J. Differ. Equ., 252 (2012), 1169-1181. 
[35] S. Zhang, C. Liu and H. Zhang, Numerical simulations of hydrodynamics of nematic liquid crystals: Effects of kinematic transports, Commun. Comput. Phys., 9 (2011), 974-993.

[36] Q. Zhang, H. Su and X. Feng, A partitioned finite element scheme based on Gauge-Uzawa method for time-dependent MHD equations, Numer. Algor., 78 (2018), 277-295. 